\title{
MicroRNA-7a regulates pancreatic $\beta$ cell function
}

\author{
Mathieu Latreille,,$^{1,2}$ Jean Hausser, ${ }^{3}$ Ina Stützer, ${ }^{1,2}$ Quan Zhang, ${ }^{4}$ Benoit Hastoy, ${ }^{4}$ Sofia Gargani, ${ }^{5}$ \\ Julie Kerr-Conte, ${ }^{5}$ Francois Pattou, ${ }^{5}$ Mihaela Zavolan, ${ }^{3}$ Jonathan L.S. Esguerra, ${ }^{6}$ Lena Eliasson, ${ }^{6}$ \\ Thomas Rülicke, ${ }^{7}$ Patrik Rorsman, ${ }^{4}$ and Markus Stoffel ${ }^{1,2,8}$
}

\begin{abstract}
${ }^{1}$ Institute of Molecular Health Sciences and ${ }^{2}$ Competence Center of Systems Physiology and Metabolic Disease, ETH Zurich, Zurich, Switzerland.
${ }^{3}$ Biozentrum, University of Basel, Basel, Switzerland. ${ }^{4}$ Oxford Centre for Diabetes, Endocrinology and Metabolism, University of Oxford, Churchill Hospital, Headington, United Kingdom. 5 Université de Lille 2, INSERM, CHRU de Lille, 1, European Genomic Institute for Diabetes, Lille, France. ${ }^{6}$ Department of Clinical Sciences Malmö, Lund University Diabetes Centre, Lund University, Malmö, Sweden. ${ }^{7}$ Institute of Laboratory Animal Science, Vetmeduni Vienna, Vienna, Austria. ${ }^{8}$ Faculty of Medicine, University of Zurich, Zurich, Switzerland.
\end{abstract}

\begin{abstract}
Dysfunctional microRNA (miRNA) networks contribute to inappropriate responses following pathological stress and are the underlying cause of several disease conditions. In pancreatic $\beta$ cells, miRNAs have been largely unstudied and little is known about how specific miRNAs regulate glucose-stimulated insulin secretion (GSIS) or impact the adaptation of $\beta$ cell function to metabolic stress. In this study, we determined that miR-7 is a negative regulator of GSIS in $\beta$ cells. Using Mir7a2 deficient mice, we revealed that miR-7a2 regulates $\beta$ cell function by directly regulating genes that control late stages of insulin granule fusion with the plasma membrane and ternary SNARE complex activity. Transgenic mice overexpressing miR-7a in $\beta$ cells developed diabetes due to impaired insulin secretion and $\beta$ cell dedifferentiation. Interestingly, perturbation of miR-7a expression in $\beta$ cells did not affect proliferation and apoptosis, indicating that miR-7 is dispensable for the maintenance of endocrine $\beta$ cell mass. Furthermore, we found that miR-7a levels are decreased in obese/ diabetic mouse models and human islets from obese and moderately diabetic individuals with compensated $\beta$ cell function. Our results reveal an interconnecting miR-7 genomic circuit that regulates insulin granule exocytosis in pancreatic $\beta$ cells and support a role for miR-7 in the adaptation of pancreatic $\beta$ cell function in obesity and type 2 diabetes.
\end{abstract}

\section{Introduction}

Type 2 diabetes (T2D) results from an inability of $\beta$ cells to secrete sufficient insulin to meet the increased metabolic demands associated with peripheral insulin resistance (1). Genetic and environmental factors as well as aging have been shown to be major risk factors for the gradual decline in insulin secretion. Genome-wide association studies have revealed a variety of $\mathrm{T} 2 \mathrm{D}$ susceptibility genes (e.g., KCNJ11, TCF7L2, and CDKAL1), many of which appear to be involved primarily in pancreatic $\beta$ cell differentiation and function (2). Several molecular pathways such as endoplasmic reticulum (ER) and oxidative stress, fatty acid and glucose toxicity, mitochondrial dysfunction, and amyloid deposition have been shown in rodent and human islets to contribute to $\beta$ cell demise and ultimately result in functional $\beta$ cell alterations and loss of $\beta$ cell mass by apoptosis $(1,3)$. While a reduction in $\beta$ cell mass is associated with T2D (4), the suppression of GSIS is more pronounced than can solely be accounted for by the loss of $\beta$ cells $(5,6)$.

RNA sequencing and expression studies have identified several microRNAs (miRNAs) expressed in mouse and human pancreatic islets (7-9). They have recently emerged as key regulators of homeostatic mechanism by profoundly influencing the responses of cells and tissues to physiological and pathological stress (10, 11). Conditional genetic deletion of dicer using Pdx1- or Ins-mediated Cre recombinase resulted in loss of insulin-producing $\beta$ cells and correlated with decreased expression of endocrine progenitor

Conflict of interest: Markus Stoffel is a scientific advisory board member of Regulus Therapeutics.

Citation for this article: J Clin Invest. 2014;124(6):2722-2735. doi:10.1172/JCI73066. markers (12). Inactivation of dicer in adult mice leads to development of severe hyperglycemia within 4 weeks after birth, resulting in glucose intolerance due to decreased insulin gene transactivation $(13,14)$. Together, these studies implicate miRNAs as positive regulators of $\beta$ cell development, survival, and insulin biosynthesis. However, only a few genetic studies have addressed the biological function of specific miRNAs in insulin-producing $\beta$ cells. We previously reported that miR-375 is a critical regulator of $\beta$ cell mass expansion in mice, as genetic deletion of Mir375 results in moderate hyperglycemia, partially phenocopying Rip-Cre dicer $1^{f / f f l}$ knockout mice (15). Nonetheless, the constant exposure of pancreatic $\beta$ cells to various metabolic stresses suggests that a delicate balance between positive and negative regulatory miRNAs likely exists in these endocrine cells to preserve their intricate identity, function, and turnover.

miR-7 is an evolutionarily highly conserved and is considered to be a prototypical neuroendocrine miRNA, being expressed at high levels in neurons and neuroendocrine organs, most notably the endocrine pancreas and the pituitary and adrenal glands $(7,16-18)$. In both invertebrate and vertebrate animals, miR-7 is coexpressed with a set of specific transcription factors that specify neurosecretory control centers of the brain (19). Such an evolutionarily ancient neuronal signature is further shaped by tissuespecific factors that restrict expression of miR-7 in non-neuronal cell types through regulating the processing of its precursor (20, 21). A novel mechanism of miR-7 regulation was recently described in neuronal cells through the identification of a brain-specific circular RNA composed of several dozens of conserved miR-7 binding sites counteracting repression by this miRNA $(22,23)$. We now 
present studies elucidating the physiological function and mRNA targets of miR-7 in pancreatic $\beta$ cells and examining its role in the context of T2D. Our results established miR-7 as the first negative regulator of insulin secretion in $\beta$ cells and revealed a miR-7regulated network interconnecting the exocytosis machinery with $\beta$ cell transcription factors driving differentiation, thus conferring functional robustness to pancreatic $\beta$ cells.

\section{Results}

Genetic deletion of Mir7a2 results in increased glucose-stimulated insulin secretion. We determined that the Mir 7 gene family was highly expressed in the pituitary gland, pancreatic islets, and hypothalamus (Supplemental Figure 1A; supplemental material available online with this article; doi:10.1172/JCI73066DS1). Mouse and human pancreatic islets displayed approximately 15 -fold higher levels of miR-7 compared with adrenal glands, while miR-7 expression was almost undetectable in the thyroid (Supplemental Figure 1, $A$ and $B$, and ref. 7). Analysis of the miR-7 precursors revealed that miR-7a2 was the most abundant member of the miR-7 family in pancreatic islets (Supplemental Figure 1C). To study the consequence of reduced miR-7a levels in pancreatic $\beta$ cells, we generated Mir7a1 and Mir7a2 conditional knockout mice using the Cre/Lox system (Supplemental Figure 2, A and B). Mutant mice were verified by Southern blotting (Supplemental Figure 2, C and D). Homozygous Mir7a1 and Mir7a2 floxed mice (Mir7a $1^{f / f l}$ and Mir $7 a 2^{f / f l}$, respectively) were crossed with Rip-Cre transgenic animals (24) to selectively ablate expression in $\beta$ cells. Assessment of recombination efficiency by the Cre transgene revealed selective deletion of miR-7 genes in pancreatic islets (Supplemental Figure 2E). Rip-Cre Mir7a1 flfl and Rip-Cre Mir7a $2^{f l / f l}$ mice were born at Mendelian frequencies and were seemingly normal. Expression analysis revealed an approximately $20 \%$ decrease in total miR-7a levels in Rip-Cre Mir7a1 ${ }^{f l f l}$ versus Mir7a1 ${ }^{f l f l}$ islets, while miR-7a expression decreased approximately $80 \%$ in Rip-Cre Mir7a $2^{f / f l}$ versus Mir7a $2^{f / f l}$ islets (Figure 1A), which demonstrated that most of the miR-7a expression in $\beta$ cells is attributable to the activity of Mir 7 a2. Importantly, expression of miR-7b was not affected by Mir7a1 or Mir7a2 gene deletions (Figure 1A), indicative of no compensation by miR-7 family members. Metabolic analysis of mice revealed similar weight, blood glucose, i.p. glucose tolerance test (IPGTT), and i.p. insulin tolerance test (IPITT) in both male and female Rip-Cre Mir7a ${ }^{f / f l}$ mice and control Mir7a $1^{f l f l}$ and Rip-Cre littermates (Supplemental Figure 2, F-I, and data not shown). In contrast, although weight and glycemia remained similar to that of littermate controls, RipCre Mir7a2 $2^{f / f l}$ mouse glucose tolerance improved when challenged in an IPGTT (Figure 1, B-D). Importantly, higher levels of insulin were measured in Rip-Cre Mir7a2flfl mice at 5, 15, and 30 minutes after glucose injection compared with control mice (Figure 1E). Insulin sensitivity was not altered in Rip-Cre Mir7a $2^{f l / f l}$ animals at 10 and 18 weeks of age (Figure 1F and data not shown). Collectively, these results indicate that deletion of Mir $7 a 2$ in $\beta$ cells improves glucose tolerance by increasing insulin secretion.

To investigate the reason for the improved glucose tolerance in Rip-Cre Mir7a $2^{f l / f l}$ mice, we analyzed endocrine $\beta$ cell mass and function. Inspection of islet architecture of Rip-Cre Mir $7 a 2^{f / f l}$ mice revealed intact endocrine cell organization (Figure 1G). Morphometric analyses did not reveal differences in pancreatic endocrine cell mass in Rip-Cre Mir7a $2^{f l / f l}$ animals compared with Mir7a $2^{f / f l}$ controls (Figure 1H and Supplemental Figure 2, J and K). To further characterize the increased insulin secretory function of Rip-
Cre Mir7a $2^{f / f l}$ mice, glucose-induced insulin secretion assays were performed in islets of 5- and 35-week-old mice (Figure 1, I and J). In both age groups, basal insulin secretion was not affected, but when evoked by $16.7 \mathrm{mM}$ glucose, insulin secretion increased 2- to 3-fold. Insulin secretion evoked by high $\mathrm{K}^{+}$(an experimental paradigm that bypasses glucose metabolism) was also enhanced approximately 2-fold in Rip-Cre Mir7a $2^{f / f l}$ versus Mir7a2 $2^{f / f l}$ islets (Figure 1J). These results demonstrated that miR-7a 2 is a negative regulator of insulin secretion and that its ablation enhances insulin secretion by an effect exerted distally at the level of insulin granule exocytosis.

miR-7 a regulates $\beta$ cell exocytosis independently of $\left[\mathrm{Ca}^{2+}\right]_{i}$ dynamics. We correlated the effects of miR-7a overexpression (adenoviral transduction; Ad-miR-7a1 and/or Ad-miR-7a2 versus Ad-GFP control) or silencing (LNA-based miR-7a inhibitors) (Supplemental Figure 3A) on insulin secretion to changes in cytosolic free $\left[\mathrm{Ca}^{2+}\right]_{\mathrm{i}}$ in response to $20 \mathrm{mM}$ glucose or membrane depolarization produced by elevated extracellular $\mathrm{K}+(35 \mathrm{mM})$. Regardless of whether miR-7a expression was increased or reduced, responses to glucose and membrane depolarization were qualitatively similar to the respective control cells (Figure 2, A and B). Thus, we concluded that proximal responses to glucose recognition and $\left[\mathrm{Ca}^{2+}\right]_{\mathrm{i}}$ influx are not altered by miR-7a expression.

To demonstrate a role for miR-7 in late-stage vesicle fusion, we applied high-resolution capacitance measurements of exocytosis. Exocytosis was evoked by voltage-clamp depolarizations of variable duration from -70 to $0 \mathrm{mV}$. The relationships between pulse length and exocytosis in control $\beta$ cells and cells overexpressing miR-7a are shown in Figure 2, C and D. Whereas overexpression of miR-7a inhibited exocytosis elicited through short physiological pulses (30-50 ms) by approximately 50\%, longer depolarizations were without inhibitory effect (data not shown). The significance of these findings is further supported by the actual duration of $\beta$ cell action potential by glucose, which ranges $30-50 \mathrm{~ms}(25)$. This inhibition of exocytosis by miR-7a occurred without any statistically significant reduction of the voltage-gated $\mathrm{Ca}^{2+}$ current. In $\beta$ cells overexpressing miR-7a, the peak $\mathrm{Ca}^{2+}$ current during a depolarization from -70 to $0 \mathrm{mV}$ averaged $-163 \pm 25 \mathrm{pA}(n=12)$, similar to the $-129 \pm 15 \mathrm{pA}(n=17)$ measured in GFP-expressing control cells (data not shown). Based on these observations, we concluded that miR-7 suppresses insulin secretion in response to glucose-induced action potential firing.

We also studied the effect of miR-7a silencing on exocytosis (Figure 2E). Exocytosis was dramatically increased in cells treated with LNA-based miR-7a inhibitors for all pulse durations compared with control cells (Figure 2F). This stimulation of exocytosis occurred without any statistically significant effect on $\mathrm{Ca}^{2+}$ current; the peak $\mathrm{Ca}^{2+}$ current during depolarizations to $0 \mathrm{mV}$ averaged $-85 \pm 16 \mathrm{pA}(n=13)$ and $-102 \pm 19 \mathrm{pA}(n=15)$ under control conditions and after inhibition of miR-7a, respectively (data not shown). Importantly, similar stimulatory effects on exocytosis were observed in human $\beta$ cells upon LNA-based miR-7a inhibition (Figure $2 \mathrm{G}$ ), which indicates that regulation of insulin granule exocytosis by miR-7a is evolutionarily conserved.

We finally used total internal reflection fluorescence microscopy to determine the number of docked/near-membrane secretory granules in $\beta$ cells exposed to $20 \mathrm{mM}$ glucose (Figure $2, \mathrm{H}$ and I, and Supplemental Figure 3B). The density of docked granules averaged approximately 1 granule $/ \mu \mathrm{m}^{2}$ in control cells and was not affected by inhibition or overexpression of miR-7a at $1 \mathrm{mM}$ glucose exposure. Similar results were observed in cells exposed to $20 \mathrm{mM}$ glu- 

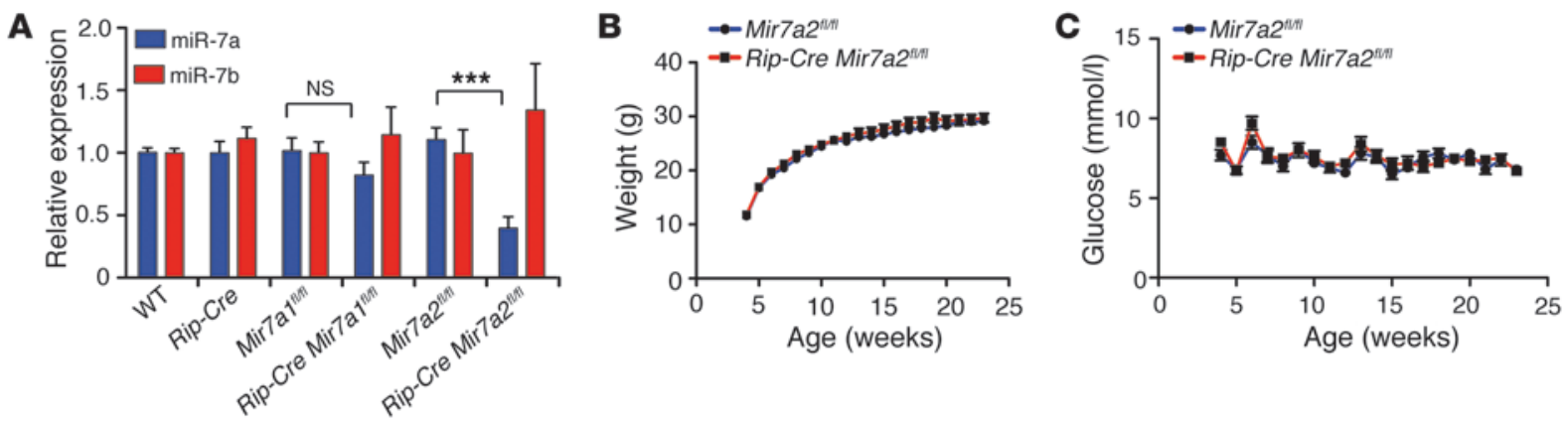

D

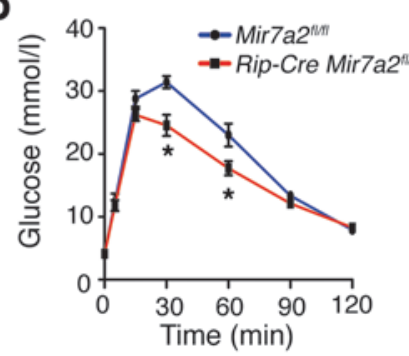

G

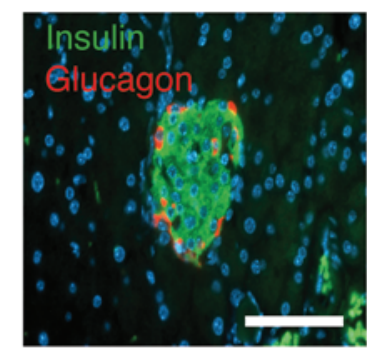

Mir7a2 ${ }^{\text {thI }}$
E
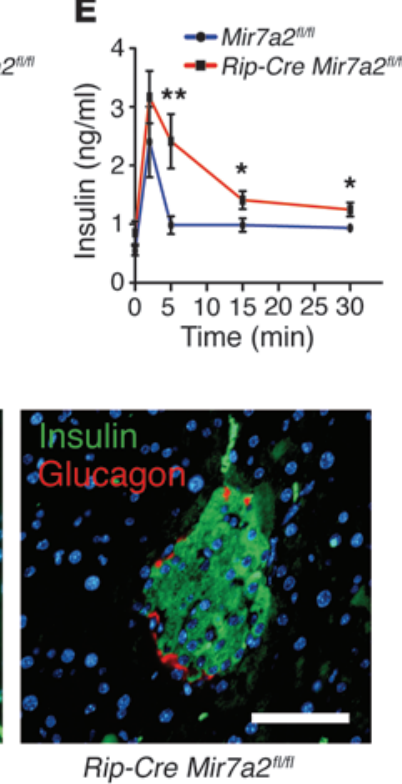

Rip-Cre Mir7a2 ${ }^{\text {thr }}$
$\mathbf{F}$

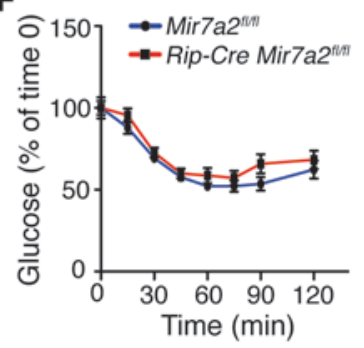

H

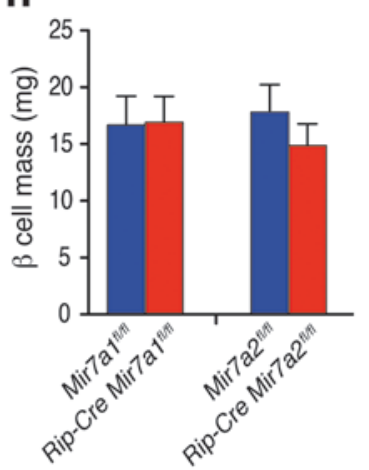

I

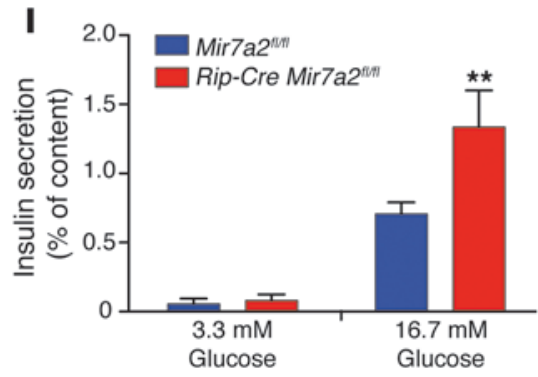

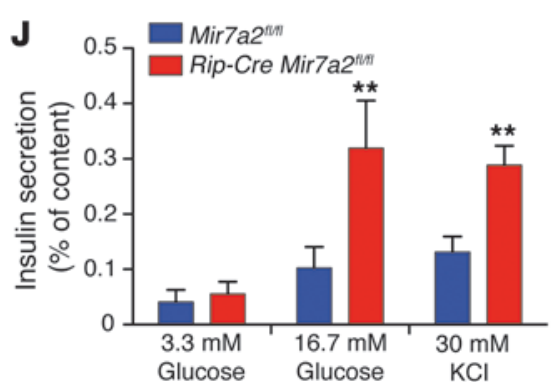

Figure 1

$\beta$ cell-specific Mir7a2 loss-of-function mouse models display increased glucose tolerance due to improved secretory function. (A) Relative miR-7a and miR-7b expression in pancreatic islets

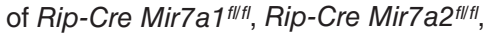
and control mice at 8 weeks of age $(n=5-6)$. (B) Body weight of Rip-Cre Mir7a2 ${ }^{\text {fllfl }}$ and control mice $(n=8-13)$. (C) Ad libitum-fed blood glucose levels in Rip-Cre Mir7a2 ${ }^{\text {fl/fl }}$ and control mice $(n=8-13)$. (D) IPGTT $(3 \mathrm{~g} / \mathrm{kg})$ in overnight fasted Rip-Cre Mir7a2 ${ }^{f / l f l}$ and control mice at 10 weeks of age $(n=11)$. (E) In vivo insulin excursions in overnight fasted Rip-Cre Mir7a2 ${ }^{f / f l}$ and controls at 15 weeks of age during an IPGTT $(n=6-11)$. (F) IPITT $(0.75 \mathrm{U} / \mathrm{kg})$ in 6-hour fasted Rip-Cre Mir7a2 ${ }^{f / f l}$ and control mice at 11 weeks of age $(n=11)$. (G) Islet organization of Rip-Cre Mir7a2 $2^{\mathrm{fl} / \mathrm{fl}}$ and control pancreas at 10 weeks of age. Shown are staining for insulin and glucagon. Scale bar: $50 \mu \mathrm{m}$. (H) Pancreatic $\beta$ cell mass in 25-weekold Rip-Cre Mir7a2 ${ }^{\mathrm{fl} / \mathrm{fl}}$ and control mice $(n=5-6)$. (I and J) Static insulin secretion performed with islets from 5- (I) and 35-week-old (J) Rip-Cre Mir7a2 ${ }^{f / f l}$ and control mice $(n=5)$ at indicated glucose and $\mathrm{KCl}$ concentrations. All data are mean \pm SEM except $\mathbf{I}$ and $\mathbf{J}$ (mean \pm SD). ${ }^{*} P<0.05 ;{ }^{* \star} P<0.01 ;{ }^{* \star} P<0.001$. cose. Mouse $\beta$ cells have a surface area of approximately $600 \mu \mathrm{m}^{2}$ (26). The above values therefore correspond to 600 docked granules per cell, in excellent agreement with data previously derived by electron microscopy (27). These observations argue that the stimulation of exocytosis after inhibition of miR-7a cannot be attributed to an increased number of docked granules. Thus, these data indicate that inhibition of miR-7a acts by increasing the release competence of granules already at the plasma membrane.

Identification of miR-7 target genes in pancreatic $\beta$ cells. To address the molecular basis for the metabolic alterations observed in miR$7 \mathrm{a} 2$ null mice, we performed unbiased genome-wide expression analyses on (a) MIN6 cells infected with Ad-miR-7a2 or Ad-GFP control and (b) pancreatic islets from Rip-Cre Mir7a $2^{f l f l}$ mice and control animals. Of 15,414 genes on the array, a total of 1,386 were downregulated at least 1.3-fold in Ad-miR-7a2-infected MIN6 cells compared with Ad-GFP-infected controls, while 1,739 genes were upregulated by the same cutoff. Transcripts that carry miR-7 motifs showed significant downregulation compared with transcripts that do not $\left(P<10^{-6}, 2\right.$-sided Mann-Whitney test; Figure 3A). The downregulation was even stronger for transcripts containing evolutionarily selected miR-7 motifs (top 100 predicted targets by EIMMo site, $P<10^{-6}$ ). These data demonstrated that direct targets of miR-7 are regulated through gene dosage and suggested that the regulatory function of miR-7 is under evolutionarily selective pressure. 
A

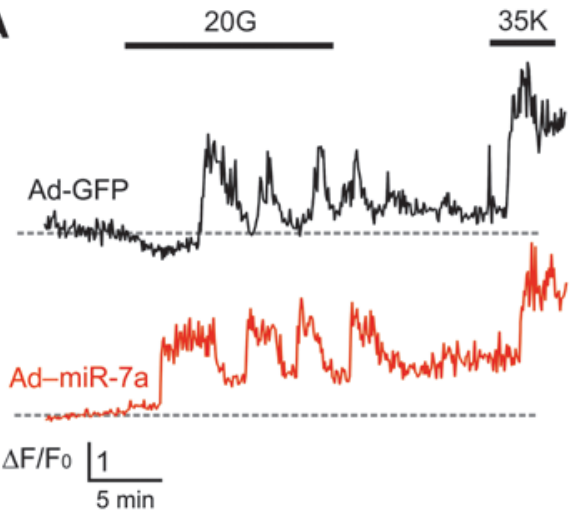

C

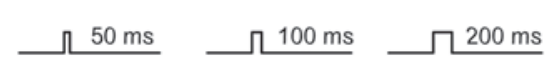

B
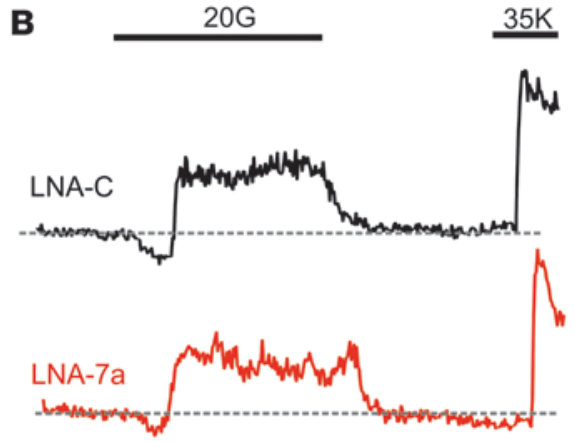

E
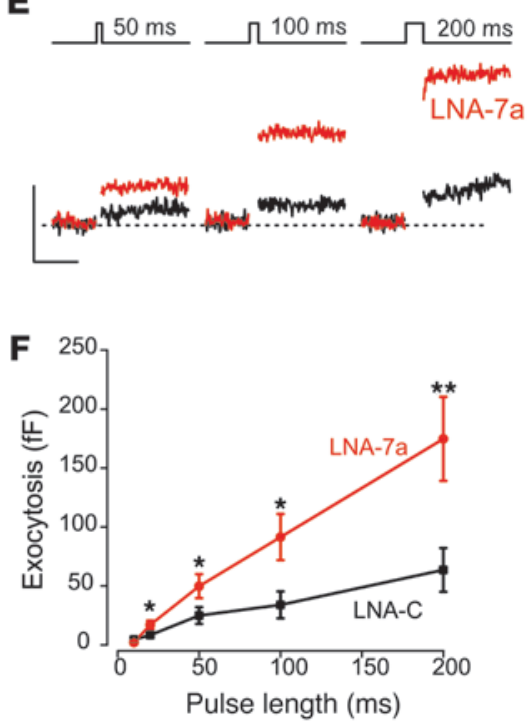

$35 \mathrm{~K}$

4 mary pancreatic $\beta$ cells. ( $A$ and $B$ ) Calcium responses in $(\mathbf{A})$ control (Ad-GFP) and miR-7a-overexpressing ( $\mathrm{Ad}-\mathrm{miR}-7 \mathrm{a})$ and (B) control (LNA-C) and miR-7a-inhibited (LNA7a) $\beta$ cells under basal conditions and after addition of $20 \mathrm{mM}$ glucose (20G) or $35 \mathrm{mM} \mathrm{KCl} \mathrm{(35K).} \mathrm{Traces}$ are representative of 40 (Ad-GFP), 22 (Ad-miR-7a), 14 (LNA-C), or 16 (LNA-7a) different cells from 3 different animals and expressed as $\Delta F / F_{0}$ (i.e., change relative to basal fluorescence [dashed horizontal lines]). (C and E) Exocytosis, monitored as increases in membrane capacitance, in (C) miR-7a-overexpressing and (E) miR-7a-inhibited $\beta$ cells. Exocytosis was elicited by progressively longer (10-500 ms) depolarization from -70 to $0 \mathrm{mV}$ (shown above traces). For clarity, only responses to 50-, 100-, and 200-ms depolarizations are shown. (D and F) Relationship between pulse duration and exocytosis (quantification of results in $\mathbf{C}$ and $\mathbf{E}$, respectively). $n=14$ (Ad-miR-7a2); 16 (Ad-GFP); 14-16 (LNA-7a and LNA-C). (G) As in F, but experiments were performed on human $\beta$ cells $(n=18-20)$. ( $\mathbf{H}$ and $\mathbf{I})$ Docked granule density in $(\mathbf{H})$ miR7a-overexpressing and (I) miR-7ainhibited cells. Data are from 15-23 cells obtained from 2 (H) or 3 (I) mice after a 15-minute incubation with 1 or $20 \mathrm{mM}$ glucose. All data are mean \pm SEM. ${ }^{\star} P<0.05 ;{ }^{\star \star} P<0.01$.

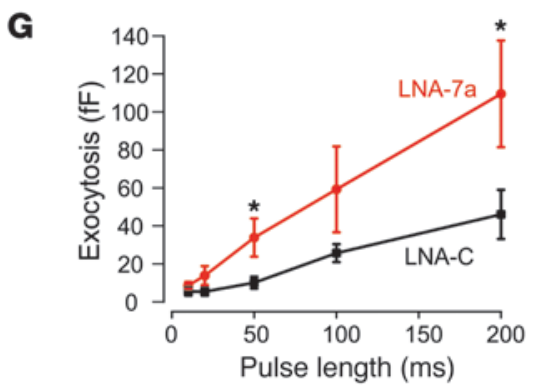

H

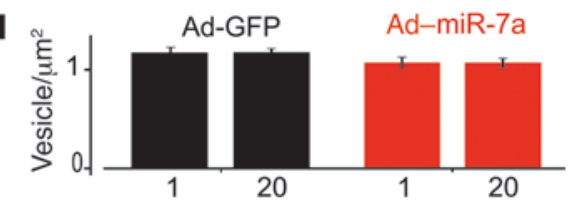

I

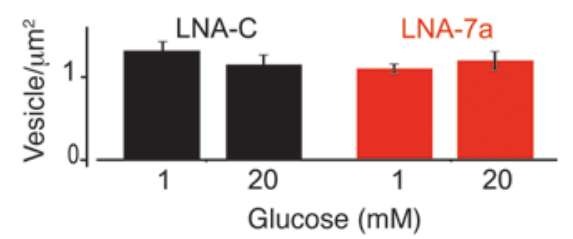

Importantly, transcriptional profiling of miR-7a1- and miR-7binfected cells also displayed a strong enrichment of miR-7 seeds in downregulated genes with striking overlapping expression profiles $(r=0.95$; data not shown), suggestive of engagement of a similar set of transcripts by all miR-7 family members. An additional array analysis performed on samples derived from Mir7a2 mutant islets revealed that 1,385 transcripts were upregulated in Rip-Cre Mir $7 a 2^{f l / f l}$ islets, 437 of which contained at least 1 miR-7 seed match. We found a strong inverse correlation between transcript levels and the presence of miR-7 seed in their $3^{\prime}$-untranslated region ( 3 '-UTR; $P<10^{-3}$; Figure 3B). Looking beyond transcripts with miR-7 sites, we observed a general inverse correlation in gene expression between the overexpression and knockout experiments $(r=-0.24$; $P<10^{-15}$; Figure 3C). This indicates that single genetic inactivation of Mir7a2 is sufficient to derepress miR-7 mRNA direct targets and reprogram islet gene expression.

Gene Ontology (GO) analysis defined specific groups of genetic interactions in miR-7a2-overexpressing MIN6 cells. The types of genes that were differentially expressed clustered in specific functional classes. Genes downregulated in Ad-miR-7a2-infected cells clustered into Membrane $(P=0.001)$, Cell-to-cell signaling $(P=0.008)$, and Secretion $(P=0.03)$ pathways (Figure $3 \mathrm{D})$. To 
A

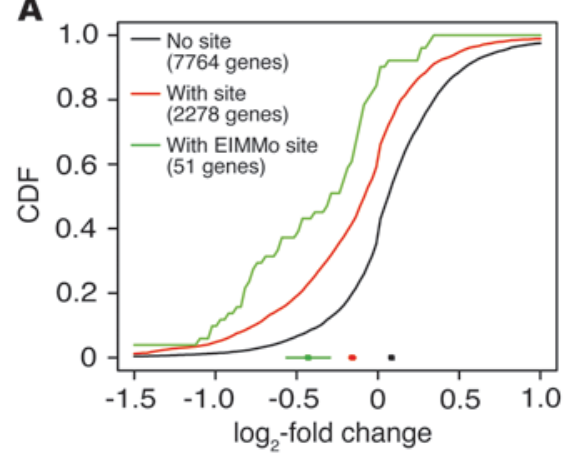

D

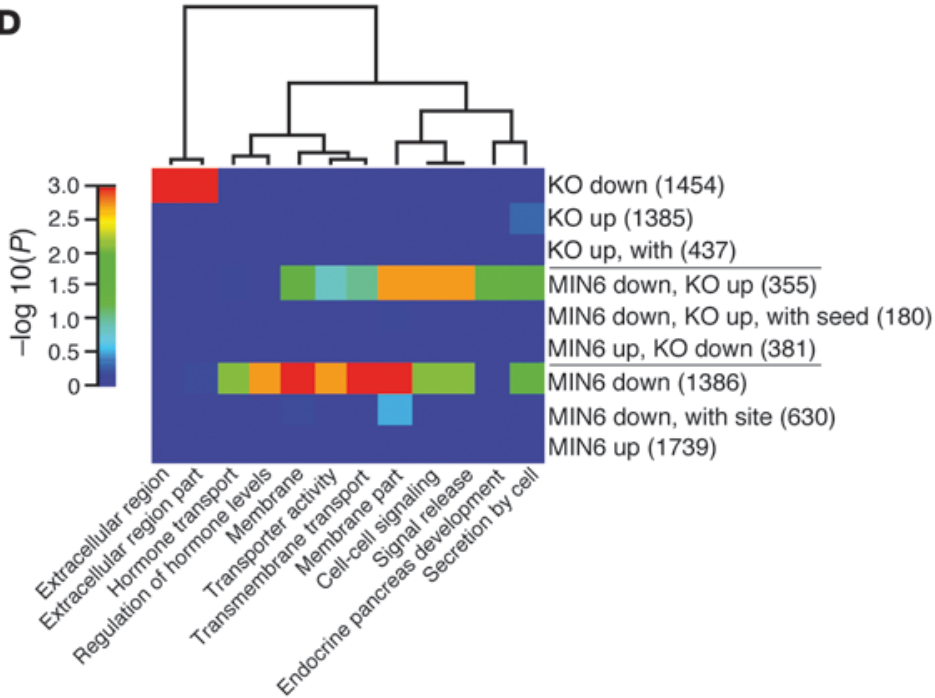

B

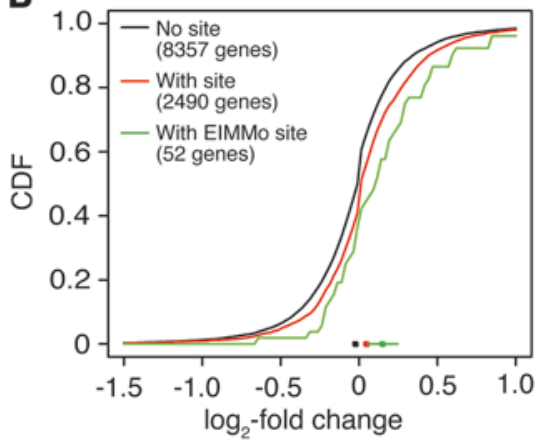

C

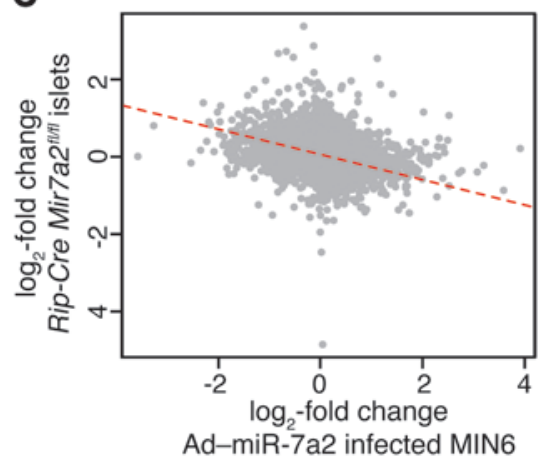

$\mathbf{E}$

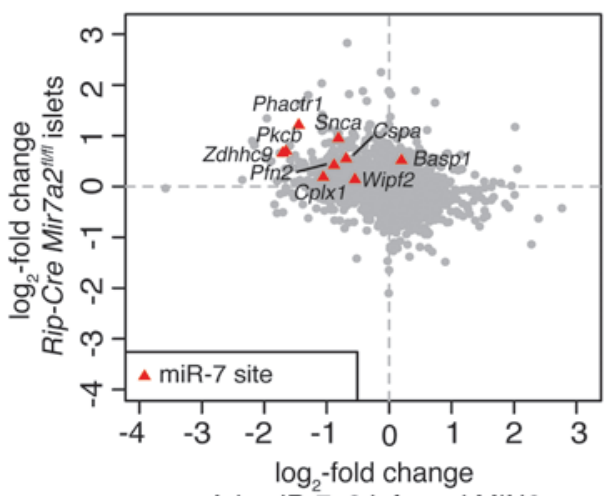

Ad-miR-7a2 infected MIN6

\section{Figure 3}

miR-7 target identification in pancreatic $\beta$ cells. (A and B) Cumulative density function (CDF) plots of $\log _{2}$-transformed gene expression fold change in (A) MIN6 cells infected with Ad-miR-7a2 vs. Ad-GFP control and (B) pancreatic islets of Rip-Cre Mir7a2 ${ }^{\text {fl/fl }}$ versus control mice. (A) $P<10^{-6}$ and (B) $P<10^{-3}$ for genes with matches to the miR-7 seed in their $3^{\prime}$-UTR (red line) and genes with matches to top EIMMo targets (green line) vs. genes lacking miR-7 seed matches (black line), 2-sided Mann-Whitney test. (C) Changes in gene expression of 9,385 genes expressed in Ad-miR-7a2-infected MIN6 cells vs. Rip-Cre Mir7a2 ${ }^{f / f l}$ islets $\left(r=-0.24 ; P<10^{15}\right.$, Pearson test). (D) GO term enrichment of genes down- and upregulated from Ad-miR-7a2-infected MIN6 cells and Rip-Cre Mir7a2 ${ }^{f / l f l}$ pancreatic islets (KO). Heatmap shows $P$ values of all significantly enriched GO terms (columns) together with the gene sets (rows) in which the terms were enriched. The number of genes in each set is indicated in parentheses. (E) Scatter plot of $\log _{2}$-transformed gene expression fold change from Ad-miR-7a2-infected MIN6 cells vs. Rip-Cre Mir7a2 ${ }^{f / f l}$ pancreatic islets within the GO classes Membrane and Secretion. Each gray dot represents a gene. Genes with miR-7 seed in their $3^{\prime}$-UTR are illustrated (red triangles).

further refine our search for the molecular basis underlying the metabolic changes occasioned by miR-7 perturbation, we analyzed genes whose expression was downregulated in Ad-miR-7a2infected MIN6 cells and upregulated in Rip-Cre Mir7a $2^{f / f l}$ islets. This analysis uncovered genes associated with similar functions (Membrane, $P=0.002$; Secretion, $P=0.024$; Cell-to-cell signaling, $P=0.002$; Figure $3 \mathrm{D}$ ). Furthermore, genes associated with the GO terms Membrane and Secretion revealed an inverse correlation in expression between the overexpression and knockout experiments (Figure 3E). Importantly, subsets of genes possessed miR-7 binding sites in their $3^{\prime}$-UTR (Figure 3E), suggestive of direct regulation by miR-7. Among them were central regulators of vesicle fusion and SNARE activity (Snca, Cspa, and Cplx1), calcium-regulated vesicular trafficking $(P k c b)$, cytoskeleton rearrangement (Pfn2, Wipf2, Basp1, and Phactr1), and membrane targeting (Zdhbc9).
We undertook an experimental validation step to confirm that miR-7 regulates the expression of genes involved in secretion and membrane dynamic in endocrine $\beta$ cells. Ad-miR-7a-mediated overexpression in MIN6 cells resulted in decreased Snca, Cspa, Cplx1, Pfn2, Wipf2, Phactr1, and Zdhbc9 transcript levels (Figure 4A). In contrast, exposure of cells to LNA-based miR-7a inhibitors increased mRNA and proteins levels of these genes (Figure 4B and Supplemental Figure 4B). Moreover, we confirmed by immunoblot analysis that overexpression of miR-7a in MIN6 cells resulted in changes of exocytotic protein levels (Figure 4C). We therefore reasoned that miR-7 targets would be derepressed in islets of Rip-Cre Mir7a $2^{R / / l}$ mice, due to decreased miRNA-mediated repression, and would contribute to increasing $\beta$ cell function of Mir7a2 mutant mice. Both mRNA and protein levels of these genes were increased in Rip-Cre Mir7a $2^{f / f l}$ islets (Figure 4, D and 

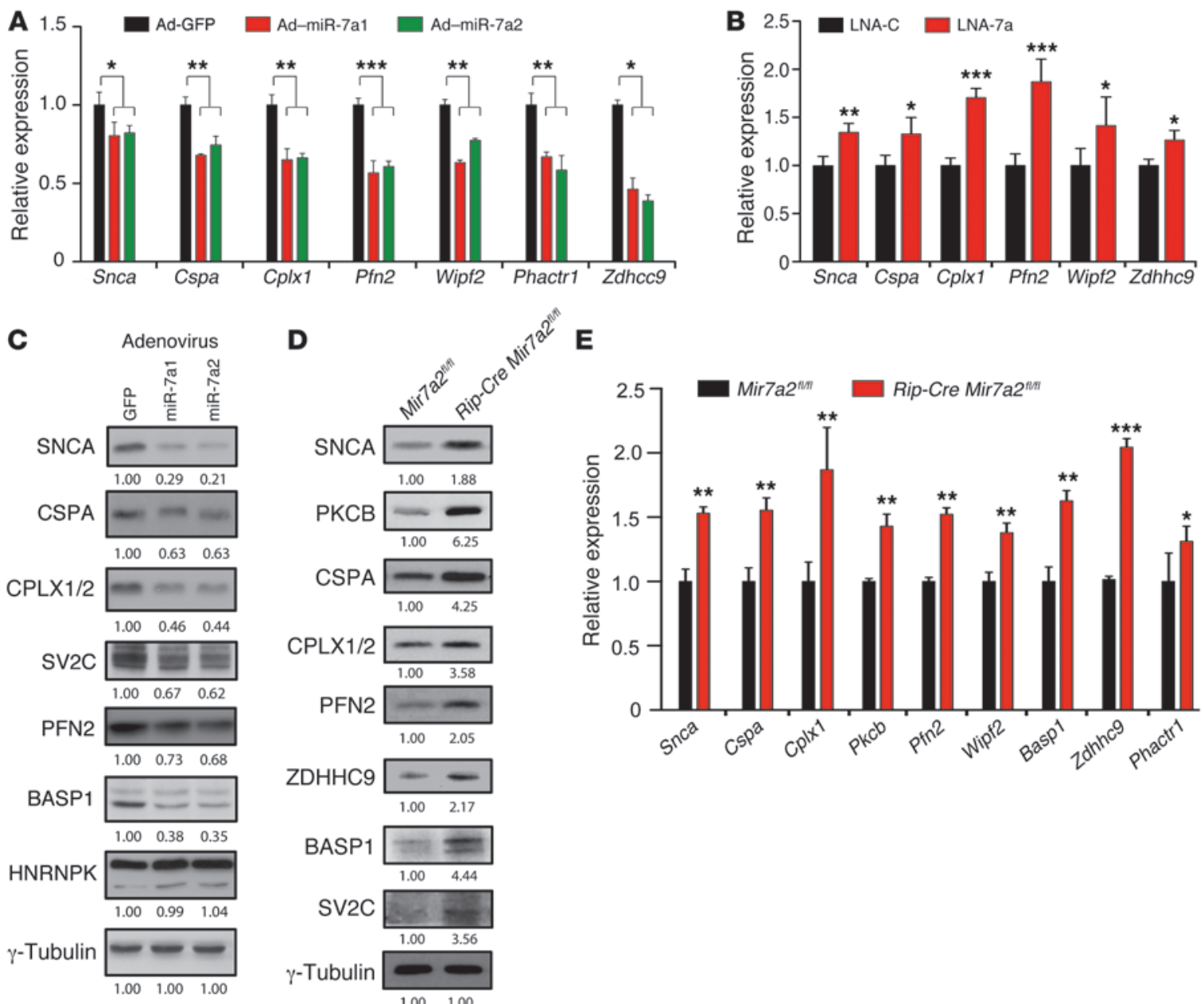

\section{E}
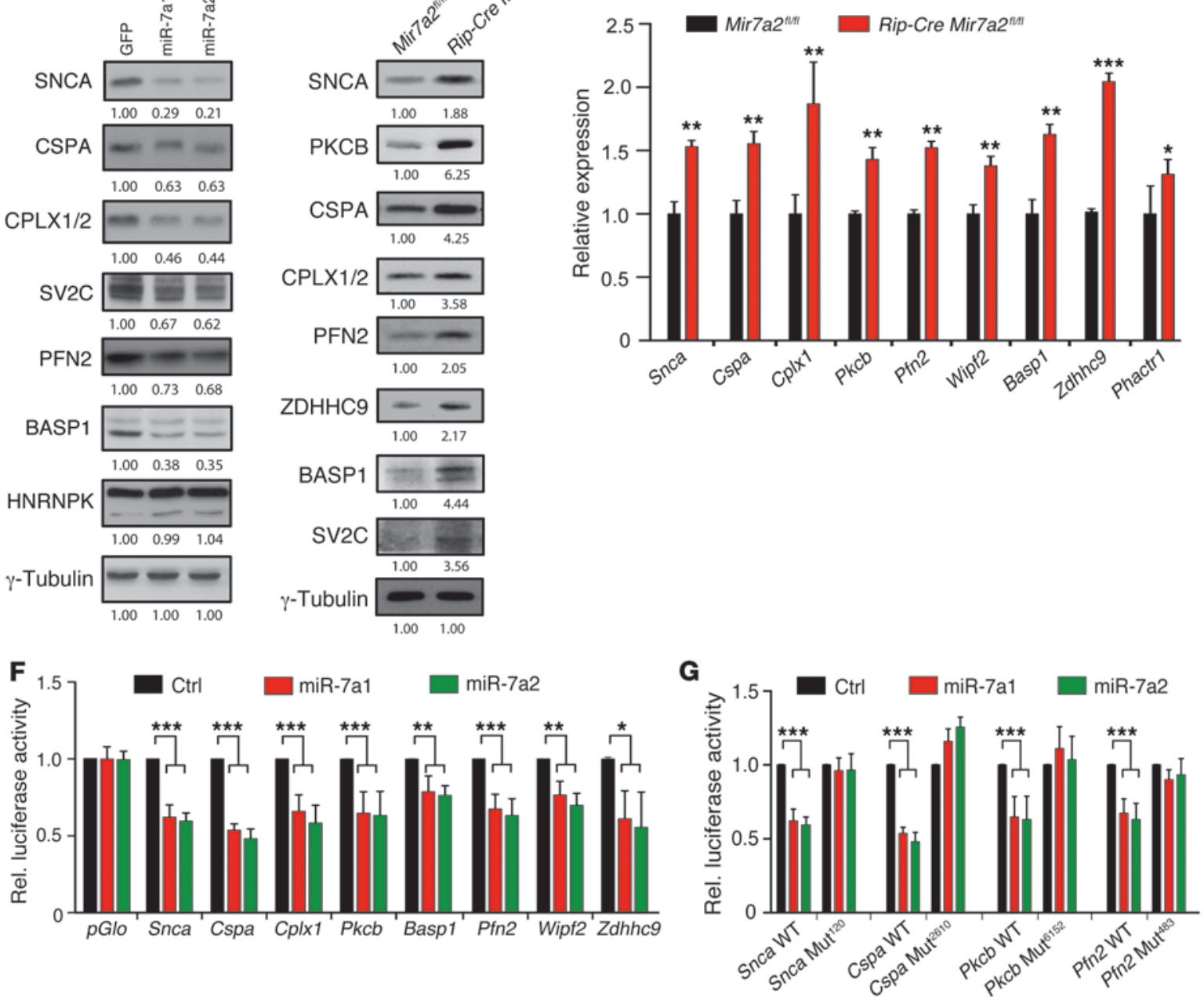

Figure 4

miR-7 represses regulators of exocytosis in pancreatic $\beta$ cells. (A and $\mathbf{B}$ ) mRNA levels of genes involved in exocytosis, in MIN6 cells either (A) infected with Ad-GFP, Ad-miR-7a1, or Ad-miR-7a2 or (B) exposed to control or miR-7a LNA inhibitors, determined by quantitative PCR $(n=3)$. (C) Western blotting analysis of MIN6 cells infected with Ad-GFP, Ad-miR-7a1, and Ad-miR-7a2. (D) Western blotting analysis of islets of control and Rip-Cre Mir7a2 ${ }^{\mathrm{flffl}}$ mice at 10 weeks of age. Islets from 5 mice per genotype were pooled, and equal fractions were loaded on SDS-PAGE gels. Protein levels normalized to $\gamma$-tubulin are indicated below. (E) mRNA levels of the indicated genes in islets of control and RipCre Mir7a2 ${ }^{\mathrm{flfl} / \mathrm{l}}$ mice at 20 weeks of age $(n=6)$. (F) Relative luciferase levels of constructs harboring $3^{\prime}$-UTRs of indicated genes in HEK293 cells cotransfected with pcDNA3 (Ctrl) and indicated pcDNA3-miR-7a vectors. Assays were performed in triplicate $(n=3-7)$. (G) Same as F, but with the indicated mutant $3^{\prime}$-UTRs. Data are mean \pm SD. ${ }^{*} P<0.05 ;{ }^{* *} P<0.01 ;{ }^{* * *} P<0.001$.

E). Our transcriptional profiling also revealed that other synaptic genes displaying positive regulatory functions in exocytosis, but lacking miR-7 seeds in their 3'-UTR, were also regulated by miR-7 in MIN6 and in islets (including $S v 2 c$; Figure 4, C and D). To test whether the effect of miR-7 on its target is mediated through the respective 3 '-UTR, firefly luciferase reporter assays were performed in cultured cells. Overexpression of miR-7a resulted in a reduction of luciferase activity associated with Snca, Cspa, Cplx1, 
Pkcb, Basp1, Pfn2, Wipf2, and Zdbhc9 3'-UTR reporters (Figure 4F). Mutations of miR-7 sites in selected targets conferred complete derepression of luciferase activity (Figure 4G and data not shown), which demonstrated that direct miRNA engagement on the 3 '-UTR of these transcripts was required for gene regulation by miR-7a. Together, these results showed that critical components of the exocytotic insulin granule machinery represent bona fide miR-7 targets in pancreatic $\beta$ cells.

miR-7 a regulates SNARE activity. Our expression analyses highlighted proteins involved in the regulation of SNARE activity and vesicle fusion as miR-7 targets. To conclusively establish that miR-7 targets enhance insulin secretion by direct modulation of the exocytotic machinery and SNARE activity, we conducted a series of biochemical analysis and secretory assays in MIN6 cells. We focused our attention on SNCA, which was recently found to promote SNARE complex assembly through an interaction with VAMP2 in neurons (28). To further demonstrate the importance of miR-7 binding to Snca 3'-UTR, primary mouse $\beta$ cells were transfected with a V5-tagged Snca expression vector fused to its natural 3'-UTR or to a 3'-UTR harboring a mutated miR-7 binding site (WT and Mut ${ }^{120}$, respectively; Figure $5 \mathrm{~A}$ ). Cells were then lysed, and levels of V5-SNCA protein were determined by immunoblotting. This analysis revealed increased levels of SNCA in Mir $7 a 2^{f / f l}$ $\beta$ cells transfected with the Mut ${ }^{120}$ compared with the WT 3'-UTR (Figure 5B). Conversely, when transfections were carried out in RipCre Mir7a $2^{f l / f l}$ cells, we measured similar levels of SNCA in both WT- and Mut ${ }^{120}$-transfected cells, which indicates that miR-7 is a critical regulator of SNCA expression through direct binding on its 3'-UTR. We then tested whether SNCA interacts with vesiclebound VAMP2 by introducing increasing amounts of V5-tagged SNCA in MIN6 cells followed by IP with a VAMP antibody. Western blotting revealed dose-dependent incorporation of SNCA in endogenous VAMP immune complexes (Figure 5C). Moreover, the amount of SNCA measured in VAMP complexes decreased in Ad-miR-7- versus Ad-GFP-infected MIN6 cells (Figure 5D). In contrast, expression of Snca Mut ${ }^{120}$ revealed increased SNCA levels recovered in immune complexes, which indicates that miR-7 regulates the amount of SNCA incorporated into VAMP complexes. SNARE oligomerization, which reflects SNARE activity, results in the formation of stable, high-molecular weight, SDSresistant oligomers and can be visualized by Western blotting under nondenaturing conditions (28). Using this assay, we found that SNCA overexpression led to a progressive increase in SNARE oligomerization, which slightly declined as SNCA levels increased, but remained above levels of control-transfected cells (Figure 5E). These results showed that the SNCA chaperoning activity is preserved in pancreatic $\beta$ cells. We then investigated whether depletion of miR-7a activity promotes SNCA-dependent SNARE oligomerization. Cells treated with LNA-based miR-7a inhibitors exhibited increased SNCA levels, which correlated with higher levels of SNAP25 in VAMP immune complexes (Figure 5F). Furthermore, LNA-based miR-7a inhibition resulted in the appearance of SDS-resistant SNARE oligomers that resolved upon boiling of the protein samples (Figure 5G). Together, these findings demonstrated that miR-7 regulates the stoichiometric interaction between SNCA and VAMP proteins and, ultimately, the equilibrium between monomeric and oligomeric SNARE complexes.

Finally, we tested whether modulation of miR-7a levels or of its targets leads to altered insulin secretion. Increased expression of miR-7a in MIN6 cells reduced both basal and glucose-induced insulin secretion (Figure 5H). Conversely, silencing of miR-7a enhanced insulin secretion upon glucose stimulation (Figure 5I), thus confirming the negative regulation of insulin secretion by miR-7a. We also studied whether change in the expression of SNCA influences the insulin secretory response of MIN6 cells. siRNA-mediated knockdown of Snca mimicked the effects of overexpressing miR-7a and decreased both basal and glucose-induced insulin secretion (Figure 5J). These data demonstrated that miR-7a controls insulin secretion by repressing expression of SNCA, culminating in the modulation of SNARE complex activity and granule fusion with the plasma membrane.

miR-7 levels regulate differentiation and $\beta$ cell function in obese mice. The improved insulin secretory phenotype of Mir7a2 knockout mice prompted us to measure miR-7a levels in islets of commonly used rodent T2D models with varying degrees of $\beta$ cell function. Samples included pancreatic islets of dietary (high-fat diet [HFD]) and genetic $(o b / o b)$ obesity mouse models on the C57BL/6N background, which displayed normoglycemia due to compensation of pancreatic $\beta$ cell function induced by insulin resistance (Supplemental Figure 5, A-C). In addition, we measured levels of miR-7a in islets of diabetic models ( $d b / d b$ mice on BKS background), which developed age-dependent hyperglycemia and reduced plasma insulin levels due to $\beta$ cell dysfunction (Supplemental Figure 5, D-F). In fact, the natural history of the BKS $d b / d b$ phenotype makes this mouse a faithful model of human obesity and $\beta$ cell failure leading to overt T2D $(29,30)$. Our analysis revealed that miR-7a levels were decreased by approximately $50 \%$ in compensating islets of HFDfed and $o b / o b$ mice (Figure 6A). We also measured significantly lower levels of miR-7a in islets from 5-week-old normoglycemic and compensating BKS $d b / d b$ mice (Figure 6B). Conversely, miR-7a expression gradually increased in decompensating BKS $d b / d b$ islets, reaching approximately 4-fold higher levels at 12 weeks of age compared with age-matched BKS controls (Figure 6B). These results revealed an unexpected inverse correlation between miR-7 levels and pancreatic $\beta$ cell function.

Since our data demonstrated that expression of miR-7a is downregulated in obese mice capable of functional islet compensation, we hypothesized that the improved metabolic control of Rip-Cre Mir $7 a 2^{f / f l}$ mice would be lost under HFD feeding due to normalization of islet miR-7a levels between obese Mir7a2flfl and lean control mice. If found to be true, this would indicate that the improved pancreatic function of lean Rip-Cre Mir $7 a 2^{f / f l}$ mice mimics that triggered by obesity and insulin resistance. Indeed, miR-7a expression in pancreatic islets of obese mice fed a HFD approached the levels measured in lean Rip-Cre Mir7a $2^{f / f l}$ animals (Supplemental Figure 6). When we exposed Rip-Cre Mir7a2fl/fl mice to HFD, we found no significant change in either weight or random-fed glycemia between obese mutant and control mice (Supplemental Figure 7, A and B). Moreover, obese controls and Rip-Cre Mir7 $2^{f f / f l}$ mice displayed similar insulin and glucose tolerance after 5, 12, and 20 weeks of HFD feeding as well as circulating insulin levels in response to glucose injection (Supplemental Figure 7, C-F, and data not shown). This finding further indicated that reprogramming of miR-7a expression in pancreatic islets of obese mice contributes to the compensatory $\beta$ cell secretory function instigated by insulin resistance and obesity.

To demonstrate the pathological significance of increased miR-7a levels in pancreatic islets of diabetic BKS $d b / d b$ mice, we generated transgenic mice with increased Mir $7 a 2$ expression in pancreatic $\beta$ cells and tested whether this recapitulates some of the defects 
A

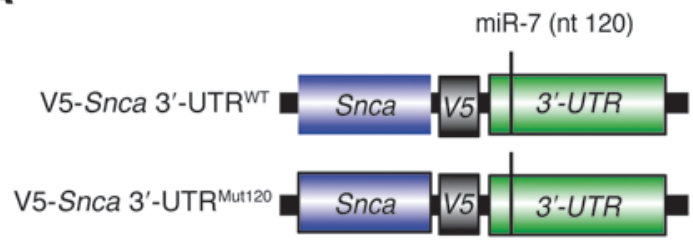

C

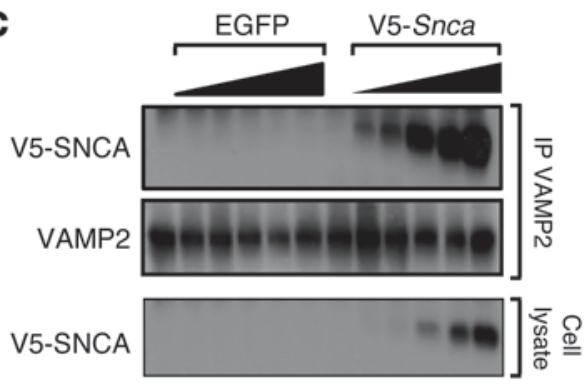

B

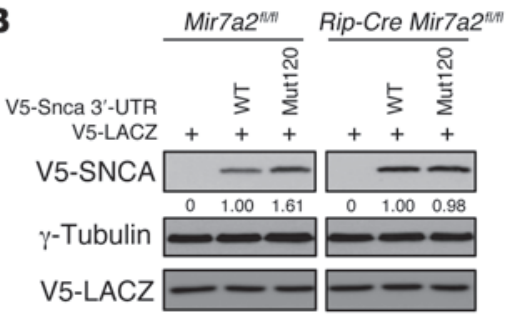

D

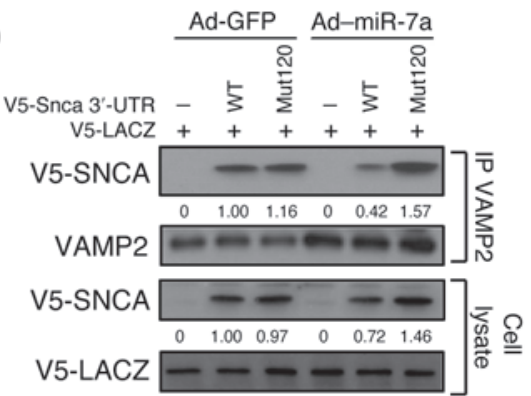

E

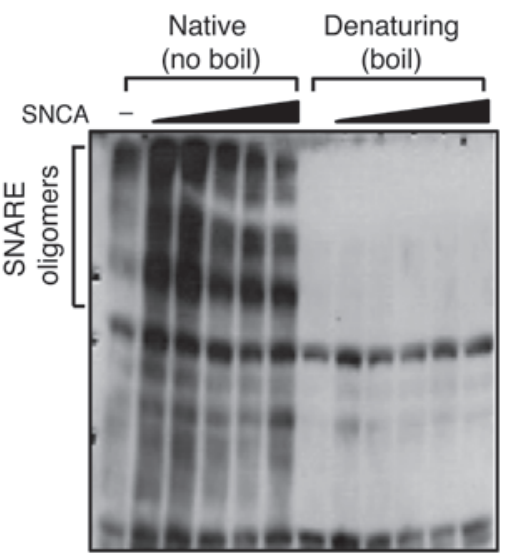

F
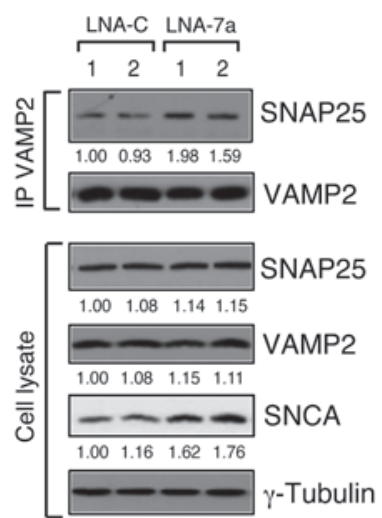

G

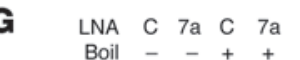

$\mathrm{kDa}$

130

100

$100-$
$70-$

$55-$

40

35

$25-$

$15^{-}$
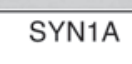

$-2-\infty$

$1.00 \quad 3.481 .40 \quad 3.68$

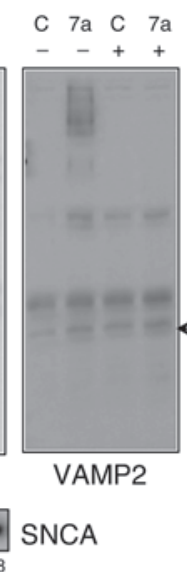

C $7 a$ C $7 a$ $-++$

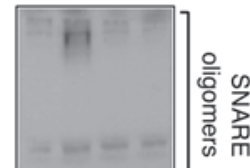

$\gamma$-Tubulin

H

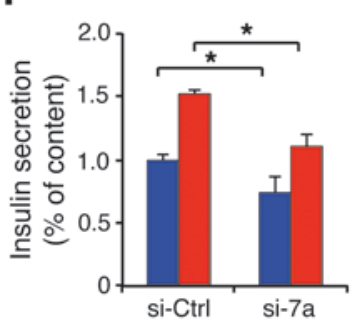

I

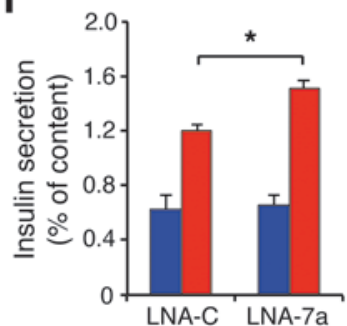

J

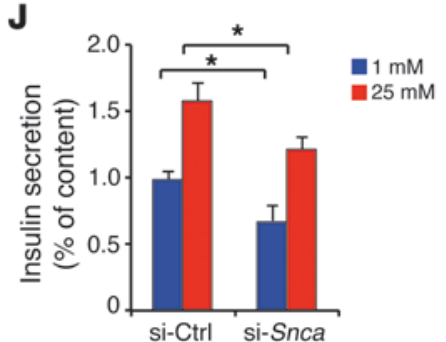

\section{Figure 5}

miR-7a and its targets regulates SNARE oligomerization and insulin secretion in pancreatic $\beta$ cells. (A) V5-tagged Snca expression vectors. (B) Dispersed islets from Mir7a2 ${ }^{f / f l}$ and Rip-Cre Mir7a2 $2^{f / f l}$ mice were cotransfected with the indicated vectors (V5-tagged Lacz served as internal control for transfection efficiency) and immunoblotted. V5-SNCA levels normalized to V5-LACZ and $\gamma$-tubulin are indicated below. (C and D) MIN6 cells were (C) transfected with V5-tagged Snca or control EGFP vectors or (D) infected with Ad-miR-7a2 and cotransfected with V5-tagged Lacz and Snca 3'-UTR WT or Mut ${ }^{120}$ plasmids, and levels of V5-SNCA in VAMP2 IPs were determined by immunoblotting. Relative V5-SNCA levels in cell lysate (normalized to V5-LACZ) and VAMP2 IP (normalized to IP VAMP2) are indicated below in D. (E) SNARE oligomerization in MIN6 cells cotransfected with VAMP2-, SNAP25-, and SYN1A-encoding vectors and increasing amounts of V5-SNCA. Immunoblotting was performed in native and denaturing conditions. (F) MIN6 cells were transfected with LNA-based miR-7a inhibitor or control, and SNAP25 was measured in VAMP2 IP. Relative levels of indicated proteins normalized to V5-LACZ and tubulin (cell lysate) or VAMP2 (IP VAMP) are indicated below. (G) MIN6 cells were transfected with LNA-based miR-7a inhibitor or control, and SNARE oligomers were detected by immunoblotting. SNCA level (bottom) served as a control. (H-J) Insulin secretion assays in MIN6 cells transfected with control or miR-7a mimics (H), miR-7a inhibitors (I), or Snca siRNAs $(\mathbf{J})(n=6)$. Data are mean \pm SD. ${ }^{*} P<0.05$. 


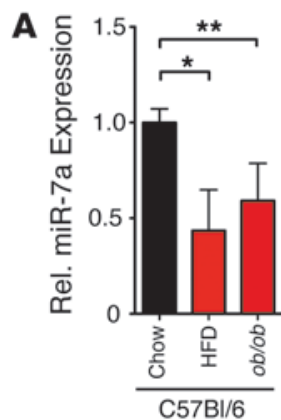

D

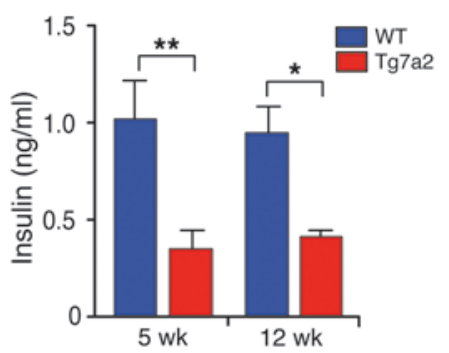

G

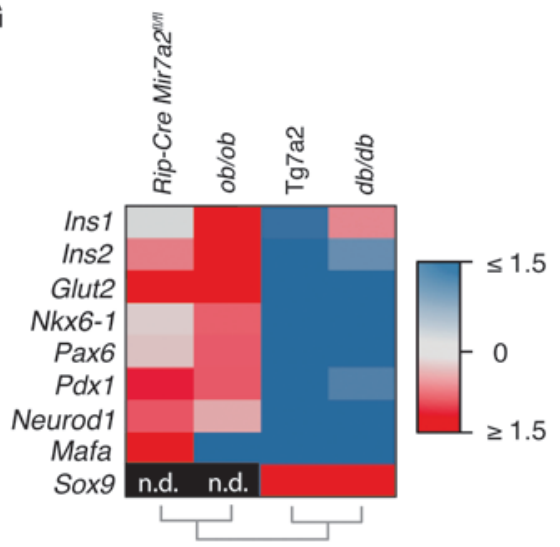

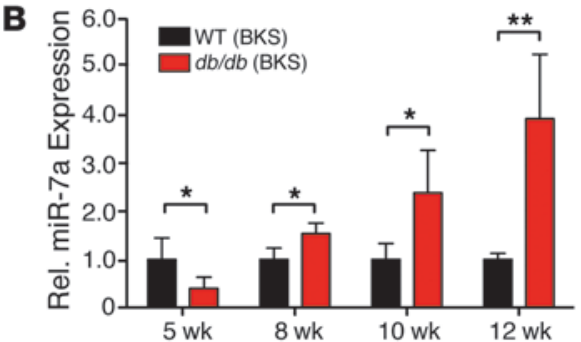
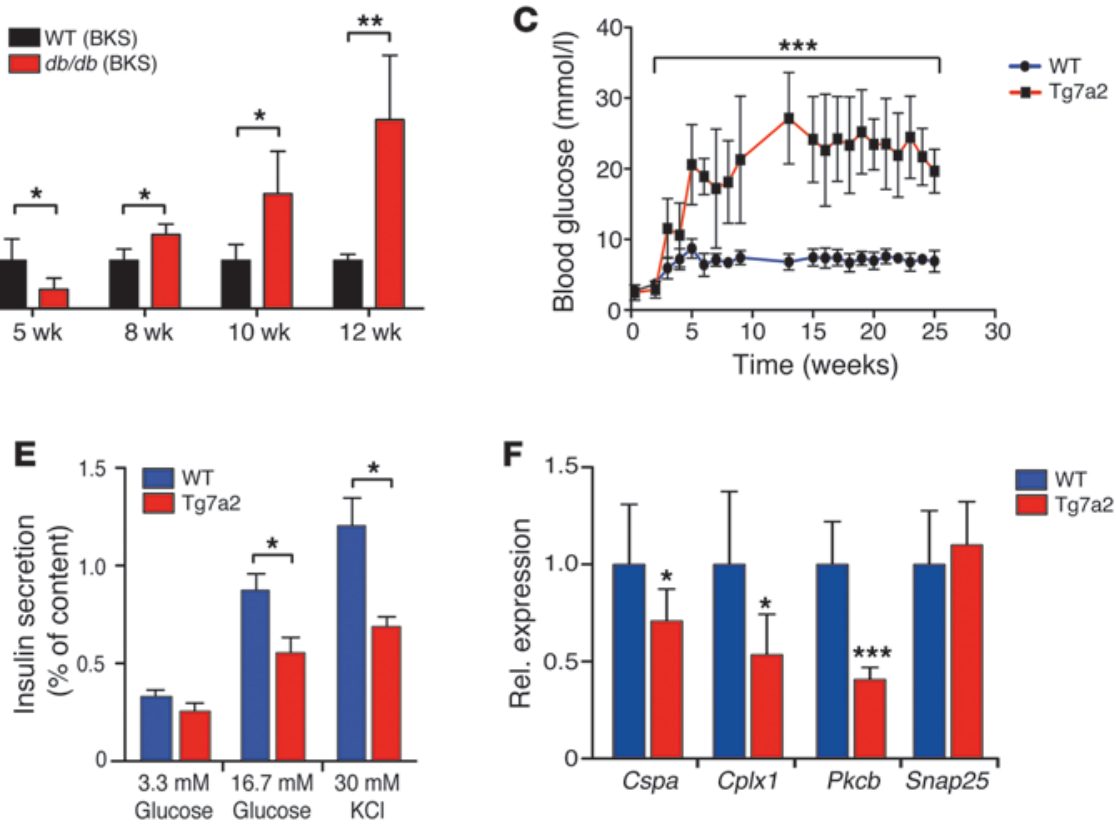

H
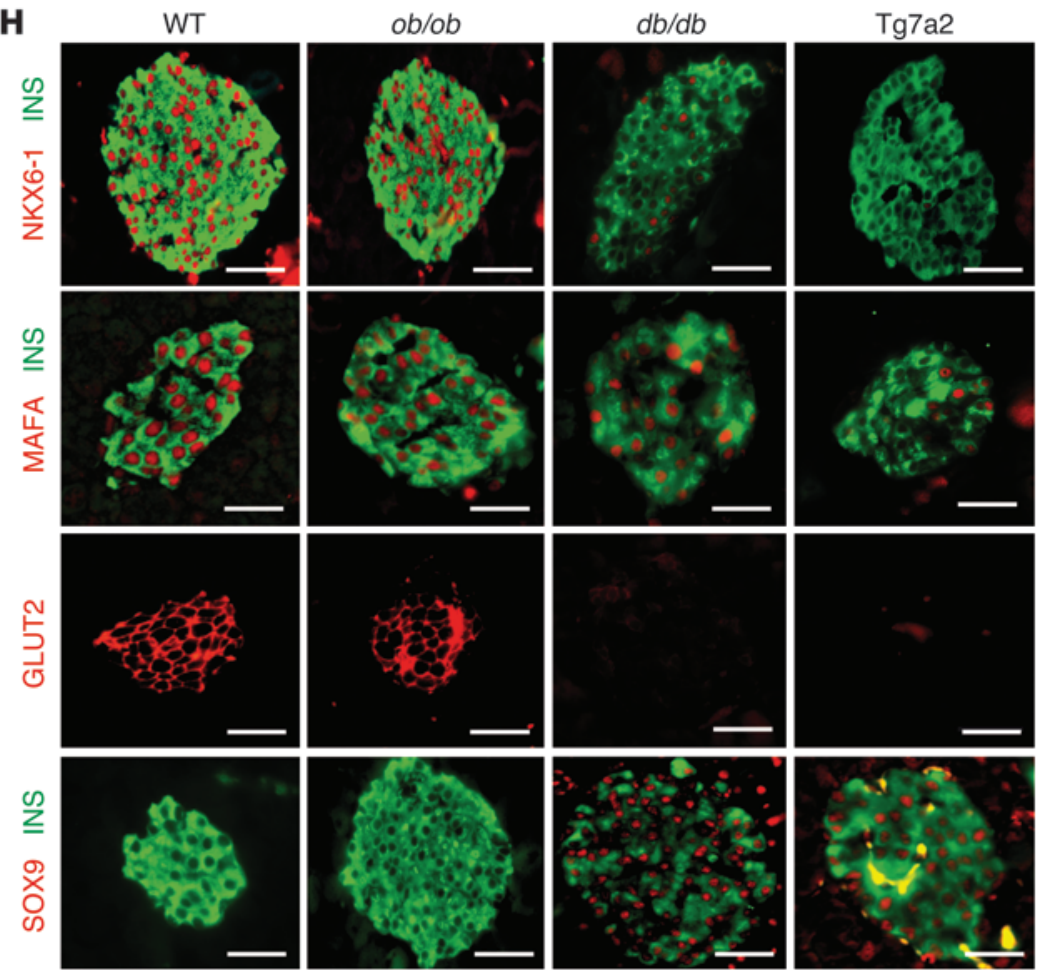

Figure 6

miR-7a levels correlate with $\beta$ cell secretory function in insulin resistant and diabetic mouse models. (A and $\mathbf{B})$ Pancreatic islets of mouse models of $\beta$ cell compensation. (A) Relative miR-7a expression of 24-week-old male mice maintained on chow or HFD and 22-week-old male ob/ob mice $(n=4-5)$. (B) Relative miR-7a expression in islets of BKS $d b / d b$ and WT control mice at the indicated ages $(n=5)$. (C) Ad libitum-fed blood glucose levels in Tg7a2 and littermate WT mice $(n=12-14)$. (D) Serum insulin levels in Tg7a2 and control mice $(n=5-6)$. (E) Insulin secretion assays performed with islets of 12-week-old Tg7a2 and control mice $(n=5)$. (F) Relative mRNA levels of exocytotic regulators in islets of Tg7a2 and control mice at 7 weeks of age $(n=5-6)$. (G) Average gene expression in islets of Rip-Cre Mir7a2 ${ }^{f / l f l}, o b / o b$, $\mathrm{Tg} 7 \mathrm{a} 2$, and BKS $d b / d b$ mice, measured by quantitative PCR ( $n=5-7$ per group). Red and blue denote increases and decreases, respectively, in gene expression vs. respective controls; no difference in expression is depicted in white. ND, expression not detectable. Hierarchical clustering analysis showing the distance or similarity between the fold changes among mouse models was performed using unweighted pair group method with arithmetic mean (UPGMA). (H) Coimmunostaining of indicated proteins in mouse models at 15 weeks of age. Scale bar: $50 \mu \mathrm{m}$. Data are mean $\pm \mathrm{SD} .{ }^{*} P<0.05 ;{ }^{* *} P<0.01 ;{ }^{* * *} P<0.001$. 
A
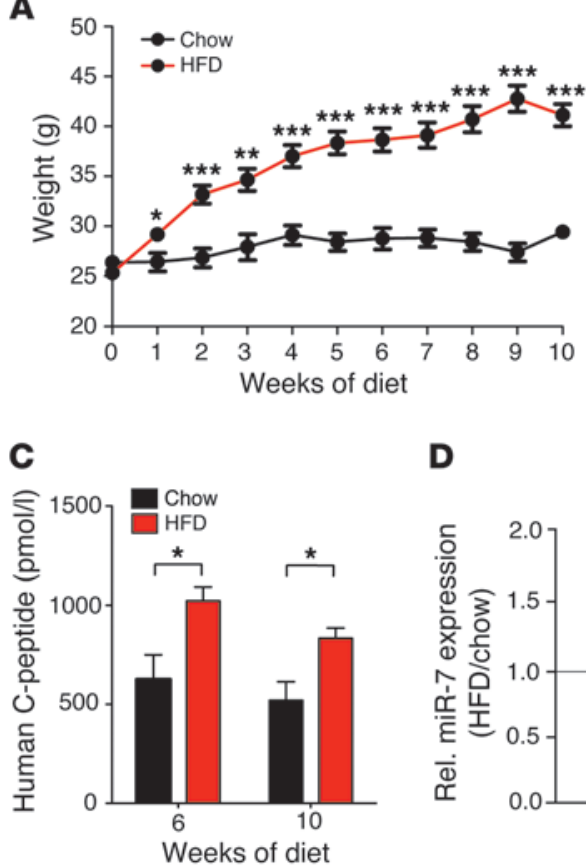

D
B
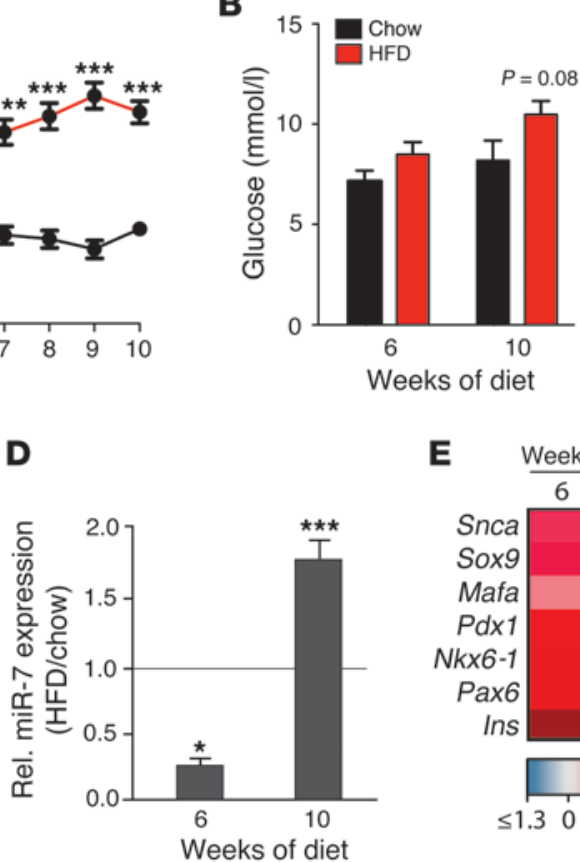

E Weeks of diet

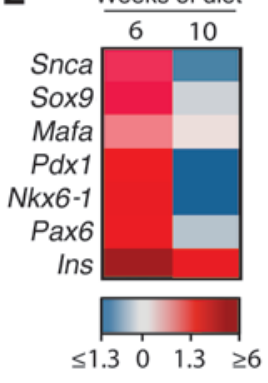

\section{Figure 7}

Adaptive changes of miR-7a levels in human pancreatic islets exposed to an obesogenic environment in the mouse. (A) Body weight of mice grafted with human islets and maintained on chow or HFD ( $n=9$ per group). (B and C) Fasting blood glucose (B) and human C-peptide (C) levels of mice grafted with human islets and maintained on chow or HFD mice for 6 and 10 weeks. (D) Normalized miR-7a expression in transplanted human islets in recipient chow- and HFD-fed mice at 6 and 10 weeks. (E) Hierarchical clustering analysis showing the distance or similarity between the fold changes in gene expression of transplanted human islets in HFDand chow-fed recipients, using unweighted pair group method with arithmetic mean (UPGMA), at 6 and 10 weeks ( $n=3$ per group). Data are mean \pm SEM. ${ }^{\star} P<0.05$; ${ }^{* *} P<0.01 ;{ }^{* \star \star} P<0.001$. observed in BKS $d b / d b$ animals. We obtained 3 founder lines with approximately 3 -fold overexpression, and founder F42 - designated as $\mathrm{B} 6 \mathrm{~N}-\mathrm{Tg}(\mathrm{Rip}-7 \mathrm{a} 2)$ and referred to herein as $\mathrm{Tg} 7 \mathrm{a} 2$ - was used for detailed analysis (Supplemental Figure 8A). Interestingly, the $\mathrm{Tg} 7 \mathrm{a} 2$ line developed marked hyperglycemia, reduced plasma insulin levels, and impaired GSIS (Figure 6, C-E). Importantly, $\alpha$ and $\beta$ cell mass as well as markers of $\beta$ cell proliferation and apoptosis were similar in $\mathrm{Tg} 7 \mathrm{a} 2$ and control littermates at 5 and 15 weeks of age (Supplemental Figure 8, B-E). Moreover, the compromised $\beta$ cell function of $\mathrm{Tg} 7 \mathrm{a} 2$ mice correlated with reduced islet $C s p a, C p l x 1$, and $P k c b$ expression levels. (Figure $6 \mathrm{~F}$ and Supplemental Figure 8F). Interestingly, pancreatic islets of Tg7a2 mice also exhibited decreased Ins1 and Ins2 levels, which correlated with a decline in expression of several transcriptional regulators preserving $\beta$ cell maturity and differentiation state, including Pdx1, Nkx6-1, Mafa, Pax6, Gata6, and Neurod1 (Supplemental Figure 8G and data not shown). We also validated that Pax6 and Gata 6 were directly repressed by miR-7a through binding to highly conserved sites within their 3'-UTRs (Supplemental Figure $8 \mathrm{H}$ and ref. 31). Together, these results demonstrated that $\beta$ cell miR-7a overexpression compromises the function and differentiation states of $\beta$ cells and, in turn, partially recapitulates metabolic features of diabetic $d b / d b$ mice.

The negative correlation between miR-7 expression and $\beta$ cell function prompted us to perform a cluster analysis of key genes maintaining $\beta$ cell identity in islets of $o b / o b$ (C57BL/ 6 background) and Rip-Cre Mir7a $2^{f l / f l}$ mice, which exhibited reduced miR-7a levels and improved insulin secretion, and in islets of BKS $d b / d b$ and Tg7a2 mice, which displayed increased miR-7a levels and impaired insulin secretion. Interestingly, the gene expression profile of RipCre Mir $7 a 2^{f l f l}$ islets was remarkably analogous to compensating $o b / o b$ islets, whereas the molecular signature of $\mathrm{Tg} 7 \mathrm{a} 2$ islets was almost indistinguishable from BKS $d b / d b$ islets (Figure 6G). Indeed, steady-state Ins1, Ins2, Pdx1, Glut2, Pdx1, Pax6, Nkx6-1, Neurod1, Mafa, and Glut2 transcript levels were increased in $o b / o b$ and Rip-
Cre Mir7a $2^{f / f l}$ islets compared with controls, but were detected at lower levels in both BKS $d b / d b$ and Tg7a2 islets (Figure 6G). These results were further validated by NKX6-1, MAFA, and GLUT2 immunostaining (Figure 6H). Interestingly, analysis of progenitor cell development markers revealed that SOX9 was induced in islets of Tg7a2 and BKS $d b / d b$ mice, but was undetectable in RipCre Mir $7 a 2^{f l / f l}$ and $o b / o b$ islets (Figure 6, G and H), which indicates that miR-7 induction triggers dedifferentiation of postnatal $\beta$ cells into a subtype with progenitor-like features. Together, these data showed that misexpression of miR-7a contributes to impairing the maturity and differentiation state of pancreatic $\beta$ cells.

To determine whether miR-7 levels correlate with the diabetogenic state in human islets, we used a transplantation model in which healthy human islets are grafted under the kidney capsule of mice maintained either on chow or on HFD to induce $\beta$ cell failure. In this experimental setting, human transplants exposed to an obesogenic environment for 12 weeks display clear signs of $\beta$ cell failure, with significantly higher glucose and lower circulating human C-peptide levels (32). We confirmed that mice fed a HFD for 6 weeks displayed early signs of insulin resistance, including increased body weight and similar blood glucose and human C-peptide level (Figure 7, A-C). Importantly, the metabolic profile of obese mice worsened over time: after 10 weeks of diet, mice maintained on HFD tended to show increased glucose levels and lower fasting human C-peptide levels compared with obese mice maintained on HFD for 6 weeks (Figure 7, A-C, and ref. 32). This obesogenic environment resulted in time-dependent change in the expression of several genes in transplanted human islets. Levels of miR-7 were more than $50 \%$ downregulated in HFD grafts at 6 weeks of diet, but increased after 10 weeks compared with chow diet transplants (Figure 7D), correlating with the diabetic/decompensation phenotype of these mice. Importantly, we found an inverse correlation between levels of miR-7 and 2 of its targets, Snca and Pax6, as well as markers of $\beta$ cell maturity (Insulin, $P d x 1, N k x 6-1$, and Mafa) and Sox9 in HFD-fed human transplant recipients 
compared with lean mice (Figure 7E). Lastly, we measured miR-7 levels in human islets from cadaver donors of matched control, obese nondiabetic, and mildly T2D individuals in 2 independent small cohorts (Supplemental Figure 9, A and B, and Supplemental Tables 1 and 2). As expected, we found lower miR-7 levels in obese and nondiabetic subjects, supporting our findings made in mouse models of $\beta$ cell compensation. On the other hand, quantitative analysis of moderate T2D human cohorts also revealed lower miR-7 levels compared with healthy controls, which suggests that these diabetic patients are in a transitory phase of the disease, mirroring obesity but preceding overt $\beta$ cell failure. However, our observations support an evolutionarily conserved role for miR-7a in the adaptive functional response of $\beta$ cells in obesity, with implications for the etiology of human T2D.

\section{Discussion}

Understanding the basic mechanism regulating the secretory response of pancreatic $\beta$ cells to glucose remains a fundamental research topic for the elaboration of novel therapeutic avenues enhancing insulin secretion and improving metabolic control of $\mathrm{T} 2 \mathrm{D}$ patients. Here, we revealed that miR-7a is a fundamental regulator of insulin secretion in pancreatic $\beta$ cells, whose expression was dysregulated in islets of mouse models of obesity and in human T2D. Using genetic approaches, we showed that conditional deletion of Mir7a2, the most highly expressed miR-7 family member in $\beta$ cells, resulted in improved glucose tolerance by increasing $\beta$ cell secretion of insulin (Figure 1). Capacitance measurements demonstrated that miR-7a inhibition augmented insulin granule exocytosis in primary mouse and human $\beta$ cells by either glucose or $\mathrm{KCl}$, a general depolarizing agent (Figure 2), thus indicating that miR-7 elicits a distal regulatory effect independent of glucose utilization. The fact that inhibition of miR-7a stimulated exocytosis by depolarizations as short as $20 \mathrm{~ms}$ also excludes the possible involvement of newcomer granules (33). If such granules contributed to the responses, they would have to move at speeds as high as $10 \mu \mathrm{m} / \mathrm{s}$, almost an order of magnitude faster than the quickest granule movements reported to date (34). Thus, miR-7a occurs to modulate the release competence of insulin granules already in place. Importantly, this effect could not be accounted for by enhanced $\mathrm{Ca}^{2+}$ entry, $\left[\mathrm{Ca}^{2+}\right]$, or the number of docked granules, thus establishing the importance of miR-7 in the regulation of insulin granule fusion with the plasma membrane. No alteration in endocrine $\beta$ cell mass was measured in Rip-Cre Mir7a $2^{f l / f l}$ mice, which showed that enhanced insulin secretion by pancreatic $\beta$ cells accounts for the phenotype of Mir7a2 mutant mice. However, this is in contrast to what Wang et al. previously showed in anti-miR-7treated $\beta$ cells, which displayed improved replicating capacity in vitro (35). The discrepancy may be explained by the promiscuity of miRNA inhibitors toward other miR-7 family members in vitro, or the masking of $\beta$ cells' growth-promoting effects by circulating hormones in vivo upon miR-7 inactivation. Characterization of complete miR-7-null mice should help resolve this issue.

Transcriptional profiling of Rip-Cre Mir $7 a 2^{f l / f l}$ pancreatic $\beta$ cells revealed that several genes possessing conserved miR-7 seeds in their 3'-UTRs were deregulated and displayed positive effects on vesicle exocytosis in neuroendocrine cells (Figure 3). Among these were Snca, Cspa, Pkcb, Cplx1, Pfn2, Wipf2, and Basp1. Snca, the gene associated with sporadic and familial Parkinsonism (36), interacts with vesicular VAMP2 and acts as a molecular chaperone promoting SNARE complex assembly (28). Likewise, deletion of the SNARE chaperone Cspa in mice causes fulminant neurodegeneration, which can be rescued by transgenic overexpression of Snca (37). We demonstrated that the SNARE chaperone activity of SNCA is preserved in pancreatic $\beta$ cells and that regulation of Snca by miR-7 ultimately determines the amount of SNCA that associates with VAMP proteins and SNARE ternary complex formation. We propose that miR-7 acts as a rheostat for SNARE activity and insulin granule fusion with the plasma membrane through modulation of SNCA levels. These data are in accordance with Steneberg et al., who revealed severe insulin secretion defects and glucose intolerance in Snca null mice (38). $\mathrm{Ca}^{2+}$-activated PKC regulators are actively recruited to the membrane compartment in primary islet $\beta$ cells in response to glucose (39), leading to the phosphorylation of several exocytotic proteins (such as SNAP25 and MUNC18) necessary for vesicle fusion and pool replenishment (40-42). Here we found that PKCB is a novel miR-7 target, which could mediate at least part of the effects of miR-7 on insulin secretion. Thus, increased expression of several exocytotic regulators contributes to improving insulin secretion in Rip-Cre Mir7a $2^{f / f l}$ mice. In this context, miR-7 represents a unique miRNA molecule for its ability to concomitantly modulate expression of several exocytotic proteins enhancing the release competence of secretory granules, thereby conferring secretory robustness to pancreatic $\beta$ cells.

Limited information exists on islet miRNA expression in chronic metabolic stress situations. We here report an inverse correlation between islet miR-7 expression and $\beta$ cell secretory function in several mouse models of T2D. miR-7 was downregulated in islets of $o b / o b$ mice that maintained normoglycemia through compensatory insulin secretion, whereas miR-7 expression was upregulated in islets of BKS $d b / d b$ mice, a diabetic model with compromised pancreatic $\beta$ cell function sharing several characteristics of human T2D (30), in accordance with the previously reported miR-7b upregulation in islets of GK rats displaying impaired GSIS (43). Interestingly, transgenic overexpression of miR-7a2 in pancreatic $\beta$ cells, occasioned chronic hyperglycemia, decreased circulating levels of insulin and impaired GSIS independent of obesity. We also detected an inverse correlation between $\beta$ cell function and miR-7 levels in transplanted human islets exposed to an obesogenic environment in mice. Indeed, miR-7 levels were decreased early upon HFD feeding in the islet compensation phase (6 weeks), but increased when islets began to decompensate (approximately 10 weeks), before marked $\beta$ cell failure dysfunction occurred at 12 weeks of age (Figure 7). miR-7 levels were also reduced in islets of obese humans subjects, which supports the notion that downregulation of miR-7 expression and derepression of its targets contribute to $\beta$ cell compensation in obesity. Surprisingly, we detected lower levels of miR-7a in islets derived from diabetic human patients (Supplemental Figure 9). HB1AC levels between healthy controls and T2D subjects (control, $5.5 \pm 0.1 \mathrm{mmol} / \mathrm{mol}$; T2D, $6.6 \pm 0.2 \mathrm{mmol} / \mathrm{mol} ; P=7.7 \times 10^{-6}$ ) were indicative of a relatively mild diabetic profile in these T2D patients, which suggests that these islets might lie in a transient functional compensatory phase of the disease or that treatment of these patients caused downregulation of miR-7 expression independently of pancreatic function.

At the molecular level, we found that diabetic Tg7a2 mice displayed downregulation of the exocytosis regulators Cspa, Cplx 1 , and $P k c b$. We also observed impaired expression of insulin and decreased levels of early (Pdx1, Pax6, and Gata6) and late (Nkx6-1, Neurod1, and Mafa) insulin gene transactivators and $\beta$ cell markers (Chga and Glut2) (44), supporting dedifferentiation of pancreatic 
$\beta$ cells by miR-7. Interestingly, deregulation of a subset of transcriptional regulators in pancreatic $\beta$ cells was previously found to underlie a fundamental cause of $\beta$ cell dysfunction leading to T2D $(30,45,46)$. Of all miR-7-regulated transcription factors, we confirmed regulation of Pax 6 by miR-7a (31) and identified Gata 6 as a novel pancreatic transcription factor directly regulated by miR-7. Both PAX6 and GATA6 have been shown to play key roles in pancreatic endocrine differentiation (31,47-50). Whereas conditional loss of PAX6 function in the adult pancreas leads to profound hyperglycemia and dedifferentiation of $\beta$ cells, with a marked reduction in PDX1, NKX6-1, and late markers such as GLUT2 and insulin (51), much less known about the molecular function of GATA 6 in adult islet $\beta$ cells, which retain significant expression levels of the transcription factor (52). Nevertheless, the sensitivity of $\beta$ cells to Gata 6 gene dosage is highlighted by heterozygous mutation in humans that can predispose to T2D development (53). Together, these findings suggest that deregulation of miR-7a represents a central cause of compromised insulin secretion and decreased expression of transcription regulators maintaining $\beta$ cell maturity. Elucidating how miR-7 expression is regulated in $\beta$ cells should help make clear how its expression impinges on the development of $\beta$ cell failure in diabetes.

An interesting observation made during the course of this study was the striking reactivation of the pre-endocrine marker SOX9 in pancreatic islets of $\mathrm{Tg} 7 \mathrm{a} 2$ and $\mathrm{BKS} d b / d b$ diabetic mice, suggestive of regression of $\beta$ cells to a developmental precursor state. The fact that multipotent SOX9-positive ductal progenitor cells can give rise to endocrine $\beta$ cells during development, but not in the adult pancreas (54), suggests that pancreatic $\beta$ cells of diabetic Tg7a2 and BKS $d b / d b$ mice may be stalled in a dedifferentiation impasse with no conceivable endocrine outcome, thereby contributing to metabolic dysfunction. Recently, Talchai et al. reported that diabetic BKS $d b / d b$ and FoxO1-null mice displayed induction of several markers strictly expressed in multipotent endocrine progenitors, including $L-M y c$, Oct4, and Ngn3, but not Sox 9 (55). However, unlike FoxO1 mutant mice, $\mathrm{Tg} 7 \mathrm{a} 2$ mice did not exhibit induction of any of these genes. Similarly, Guo et al. failed to detect induction of these genes in $d b / d b$ mice (30). Therefore, dedifferentiation of pancreatic $\beta$ cells induced by miR-7a likely proceeds through an alternative pathway, culminating in regression of $\beta$ cells to a more primitive cellular state than that in FoxO1-null mice. We propose that coinduction of miR-7a and SOX9 could represent a novel mechanism underlying pancreatic $\beta$ cell failure in diabetes. Further analyses will be required to determine whether such a mechanism also contributes to the development of pancreatic $\beta$ cell failure in humans.

Our findings revealed that changes in islet gene expression in Rip-Cre Mir7a $2^{f l f l}$ mice closely reflect molecular signatures presented by $o b / a b$ mice, which maintain lifelong robust insulin secretion. Conversely, $\mathrm{Tg} 7 \mathrm{a} 2 \mathrm{mice}, \mathrm{BKS} d b / d b$ mice, and human islet transplants exposed to HFD in mice had reduced levels of $\beta$ cell differentiation markers, thus demonstrating the significance of miR-7 upregulation in the development of $\beta$ cell dedifferentiation and failure. Our results suggest that inactivation of Mir $7 a 2$ in obese BKS $d b / d b$ mice might be sufficient to rescue $\beta$ cell failure and glycemia by enhancing $\beta$ cell maturity and pancreatic function. This was supported by our data demonstrating induction of PDX1, MAFA, and GLUT2 in Rip-Cre Mir7a $2^{f / f l}$ islets. On the other hand, we failed to identify a distinct signature for miR-7 exocytotic targets in islets of $o b / o b$ and BKS $d b / d b$ mice, as was observed in
Rip-Cre Mir7a $2^{f / f l}$ and Tg7a2 mice, which suggests that other signaling inputs can bypass the regulation of the exocytosis machinery by miR-7a. Active derepression of exocytotic regulators in RipCre Mir $72^{f / f l}$ islets indicates physical engagement of miR-7 to its targets. Hence, saturation of miR-7 binding sites on its mRNAs targets might preclude further downregulation in $\mathrm{BKS} d b / d b$ islets overexpressing the miRNA and could explain the miR-7a-independent regulation of regulators of exocytosis in these mouse models. Otherwise, obesity might provide the missing link and represent the stimulus required for reconciling expression of exocytotic regulators in these mouse models. In fact, lower levels of Snca were detected in diabetic human islet transplants exposed to HFD in mice, which supports the possibility that miR-7a-mediated repression of exocytosis regulators could underlie the gradual pancreatic dysfunctions observed in human T2D islets. Overall, our results illustrated the marked sensitivity of pancreatic $\beta$ cells to miR-7a levels and established miR-7a dysregulation as a novel contributor to pancreatic dysfunction in T2D.

The results presented herein in both mouse and human islets indicate that therapeutic inhibition of miR-7 function could be of relevance for preserving the maturity of pancreatic $\beta$ cells during the course of diabetes development by acting at 2 levels: first, by fostering the secretory capacity of $\beta$ cells through concomitant increase in the expression of several exocytotic proteins enhancing SNARE activity and insulin granule exocytosis, and second, by increasing $\beta$ cell identity via promoting expression of several key transcription factors responsible for maintaining insulin biosynthesis and differentiation states of $\beta$ cells. miR-7 inhibition might reveal a key stride toward rescuing diabetic $\beta$ cell functions, given the downregulation of several SNARE proteins previously reported in islets from diabetic mouse models $(56,57)$ and from human organ donors with T2D $(58,59)$. Lastly, many miR-7 targets identified herein have been implicated in defective neurotransmission and development of age-related neurodegenerative pathologies, such as Alzheimer and Parkinson diseases $(60,61)$. Given the common evolutionary origins of $\beta$ cells and neurons, therapeutic inhibition of miR-7a might also represent a novel approach for modulating synaptic activity and plasticity by increasing expression of exocytotic proteins required for neurotransmission. Therefore, our present study defined molecular functions of miR-7 in pancreatic $\beta$ cells, but also unveiled novel and innovating research avenues with therapeutic implications for metabolism and neurodegenerative medicine.

\section{Methods}

Experimental animals. All animal models were housed in a pathogen-free animal facility at the Institute of Molecular Systems Biology of ETH Zurich. The animals were maintained on a 12-hour light/12-hour dark cycle and had free access to either standard rodent chow or HFD containing $60 \% \mathrm{~kJ}$ from fat after weaning ( 3 weeks). All animals were on the C57BL/ 6 background except $d b / d b$ mice (BKS.(D)-Lepr ${ }^{\mathrm{db}} / \mathrm{JoelRj}$ ), which were on the BKS background (Janvier).

Antibodies. The following antibodies were used in immunohistochemistry or immunoblotting: mouse anti-V5 (Invitrogen); guinea pig anti-insulin and mouse anti- $\gamma$-tubulin (Sigma-Aldrich); rabbit anti-glucagon, rabbit anti-PDX1, and rabbit anti-SOX9 (Millipore); mouse anti-KI67 (Novocastra); rabbit anti-VAMP, mouse anti-SYN1A, mouse anti-SNAP25, rabbit anti-CPLX1/2, rabbit anti-CSPA, mouse anti-PAX6, rabbit anti-BASP1, and rabbit anti-SV2C (Synaptic Systems); goat anti-CHGA, rabbit anti-PKCB, mouse anti-PFN2, and rabbit anti-GLUT2 (Santa Cruz); goat anti-NKX6-1 
(R\&D Systems); rabbit anti-MAFA (Bethyl Laboratories); mouse anti-SNCA (BD Transduction Laboratories); rabbit anti-ZDHHC9 (Thermo Scientific).

Blood glucose measurements, IPGTT, and IPITT. Blood glucose was measured using a Contour glucometer (Bayer). For IPGTT, overnight-fasted (13 hours) mice were injected with D-glucose solution. For IPITT, animals were injected with $0.75 \mathrm{U} / \mathrm{kg}$ body weight of a $5 \times 10^{-2} \mathrm{U} / \mathrm{ml}$ insulin solution in PBS after a 6-hour fasting period.

Immunohistochemistry and morphometry. Pancreata were fixed in $4 \%$ paraformaldehyde and embedded in paraffin, and the cut sections were antigen retrieved by boiling them in $10 \mathrm{mM}$ Tris/EDTA ( $\mathrm{pH}$ 9.0). Sections were permeabilized and blocked in PBS containing $0.1 \%$ Triton X-100, $1 \%$ BSA, and $5 \%$ donkey or goat serum. Primary antibody binding was performed overnight at $4{ }^{\circ} \mathrm{C}$, while secondary antibody incubation was carried out at room temperature for 1 hour. For pancreas morphometry analysis, $8-\mu \mathrm{m}$ sections were cut, and 5 sections at least $180 \mu \mathrm{m}$ apart were taken from each mouse for each type of analysis; at least 3 mice per group were analyzed in every case. For determining $\beta$ cell and $\alpha$ cell mass, sections were stained with anti-insulin and anti-glucagon antibodies and DAPI, the pancreatic sections were scanned entirely using a $\times 10$ objective of a Zeiss AxioVert 200 microscope, and the images were recorded and assembled by AxionVision 4.6.3 software. The fraction of the insulin- or glucagonpositive areas were determined using ImageJ, and the mass was calculated by multiplying this fraction by the initial pancreatic wet weight. The fraction of KI67-positive $\beta$ cells was determined by counting at least 2,000 $\beta$ cells per mouse, as described previously (15).

Human islet transplantation model in an obesogenic environment. Experimental details of this model have been described previously (32). Nondiabetic Rag2 $2^{-/}$mice $(n=18)$ were transplanted with human islets (400 islet equivalents [IEQ]). Animals were fed for 10 weeks with control diet or HFD and followed for weight, fasting blood glucose, and human C-peptide. The groups were compared using Mann-Whitney test (non parametric). Mice were killed at the indicated time points, and cryosections of human grafts were dehydrated and microdissected (ArcturusXT Laser Capture Microdissection). RNA, extracted with PicoPure RNA extraction kit (MDS Analytical Technologies), had $\geq 6$ RNA Integrity (Agilent 2100 Bioanalyzer, Agilent Technologies); gene expression was analyzed on GeneChip Human Gene 2.0 ST Arrays (Affymetrix).

Human islets. Human islets from cadaver donors were provided by the Nordic Islet Transplantation Programme (www.nordicislets.org), Uppsala University, to the human tissue laboratory (HTL) at Lund University Diabetes Centre (LUDC). Islets were handpicked on the day of the arrival and cultured at $37^{\circ} \mathrm{C}$ for $3.4 \pm 0.5$ or $2.3 \pm 0.3$ days for controls or T2D subjects, respectively $(P=0.11)$. Donor characteristics are provided in Supplemental Tables 1 and 2. Human islets for capacitance analysis were obtained from the DRWF Clinical Islet Isolation and Transplantation Centre as previously reported (62).

Capacitance measurements. Exocytosis was monitored in isolated mouse or human $\beta$ cells by measurements of membrane capacitance essentially as reported previously (63). Briefly, depolarizations (duration 10-500 ms) from -70 to $0 \mathrm{mV}$ were applied at 30 -second intervals. The extracellular medium consisted of $138 \mathrm{mM} \mathrm{NaCl}, 5.6 \mathrm{mM} \mathrm{KCl}, 1.2 \mathrm{mM} \mathrm{MgCl}_{2}, 2.6 \mathrm{mM}$ $\mathrm{CaCl}_{2}, 5 \mathrm{mM}$ HEPES ( $\mathrm{pH} 7.15$ using $\mathrm{NaOH}$ ), and $5 \mathrm{mM}$ glucose. The standard whole-cell configuration was used, and the pipette-filling medium (dialyzing the cell interior) contained $125 \mathrm{mM}$ Cs-glutamate, $10 \mathrm{mM}$ CsCl, 10 mM NaCl, 1 mM MgCl , 0.05 mM EGTA, 3 mM Mg-ATP, 0.1 $\mathrm{mM}$ cAMP, and $5 \mathrm{mM}$ HEPES ( $\mathrm{pH} 7.1$ using $\mathrm{CsOH}$ ). In the repetitive stim- ulation experiments, 10500 -ms depolarizations from -70 to $0 \mathrm{mV}$ were applied at a frequency of $1 \mathrm{~Hz}$. The responses were measured as the increase in membrane capacitance between the prestimulatory level and the new steady-state value, once attained.

$\left[\mathrm{Ca}^{2+}\right]_{i}$ measurements. 48 hours after transfection or infection, cells were loaded for 10 minutes with $6 \mathrm{mM}$ fluo $4 \mathrm{AM}$ or $4 \mathrm{mM}$ fura redAM (Invitrogen) at $37^{\circ} \mathrm{C}$ in $1 \mathrm{mM} \mathrm{KRB}$ supplemented with $1 \mathrm{mM}$ glucose. Different $\mathrm{Ca}^{2+}$ indicators were used to avoid overlap with the transfection/infection markers. Cells were imaged on a Zeiss upright confocal LSM 510 META with $\times 40$ objective, and the excitation was triggered by $488 \mathrm{~nm}$. Emission was measured at $510-540 \mathrm{~nm}$ for fluo4AM and $>600 \mathrm{~nm}$ for fura redAM. During the experiments, cells were first exposed to $1 \mathrm{mM}$ glucose for $>15$ minutes and then exposed sequentially to $20 \mathrm{mM}$ glucose for 10 minutes, $1 \mathrm{mM}$ glucose for 15 minutes, and finally $35 \mathrm{mM} \mathrm{KCl}$ for 5 minutes.

Database repository. Gene expression profiling data were deposited in GEO (accession no. GSE48195).

Statistics. Numerical values are reported as mean \pm SD unless stated otherwise. Statistical significance was determined with a 2-tailed Student's $t$ test. A $P$ value less than 0.05 was considered significant.

Study approval. All animal experiments were approved by the Kantonale Veterinäramt Zürich and conducted in accordance with the UK Animals Scientific Procedures Act (1986) and University of Oxford ethical guidelines. All procedures related to human T2D islets were approved by the ethics committees at Uppsala and Lund Universities. Informed consent was obtained from all subjects.

\section{Acknowledgments}

We thank M. Müller, R. Kubsch, F. Núñez Villena, and K. Hermann (ETH Zurich) for technical support; K. Ahmed and D. Esterhazy for reading the manuscript; P. Lefebvre and J. Thevenet (Lille) for help with Affymetrix expression analysis and the human islet obesity mouse model, respectively; and T. Kolbe (Biomodels Austria, University of Veterinary Medicine Vienna) for pronuclei and blastocyst injections. The project was supported by The Swedish Research Council (to L. Eliasson), Region Skåne ALF (to L. Eliasson), The Swedish Diabetes Foundation (to L. Eliasson), EFSD-Lily Research Fellowship grant (to J.L.S. Esguerra), the Crafoord Foundation (to J.L.S. Esguerra), and The Knut and Alice Wallenberg Foundation. This work was supported by grants from the JDRF (Scholar grant to M. Stoffel), ERC (Metabolomirs to M. Stoffel), the Austrian Genome Research Program (GEN-AU II and III, Austromouse, to T. Rülicke), the Swiss National Science Foundation (grant no. 31003A_127307 to M. Stoffel and M. Zavolan), European Genomic Institute for Diabetes (EGID; ANR-10-LABX-46), and L'Agence de la BioMedecine and Fondation de l'Avenir (to F. Pattou and J. Kerr-Conte). Q. Zhang was supported by a Wellcome Trust Senior Investigator Award to P. Rorsman.

Received for publication September 5, 2013, and accepted in revised form March 7, 2014.

Address correspondence to: Markus Stoffel, Swiss Federal Institute of Technology (ETH Zurich), Institute of Molecular Health Sciences, Schafmattstr. 22, HPL H36, 8093 Zurich, Switzerland. Phone: 41.44.633.4560; Fax: 41.44.633.1362; E-mail: stoffel@biol.ethz.ch.

1. Kahn SE, Hull RL, Utzschneider KM. Mechanisms
linking obesity to insulin resistance and type 2 dia-
betes. Nature. 2006;444(7121):840-846.
2. Bonnefond A, Froguel P, Vaxillaire M. The emerg-
ing genetics of type 2 diabetes. Trends Mol Med.

2010;16(9):407-416.

3. Donath MY, et al. Mechanisms of $\beta$-cell death in type 2 diabetes. Diabetes. 2005;54(suppl 2):S108-S113.

4. Rahier J, Guiot Y, Goebbels RM, Sempoux C,

Henquin JC. Pancreatic $\beta$-cell mass in European subjects with type 2 diabetes. Diabetes Obes Metab. 2008;10(suppl 4):32-42

5. Porte D Jr, Kahn SE. $\beta$-Cell dysfunction and failure in type 2 diabetes: potential mechanisms. Diabetes. 2001;50(suppl 1):S160-S163. 
6. Rosengren $\mathrm{AH}$, et al. Reduced insulin exocytosis in human pancreatic $\beta$-cells with gene variants linked to type 2 diabetes. Diabetes. 2012;61(7):1726-1733.

7. Landgraf $\mathrm{P}$, et al. A mammalian microRNA expression atlas based on small RNA library sequencing. Cell. 2007;129(7):1401-1414.

8. Poy MN, et al. A pancreatic islet-specific microRNA regulates insulin secretion. Nature. 2004; 432(7014):226-230.

9. Pullen TJ, da Silva Xavier G, Kelsey G, Rutter GA. miR-29a and miR-29b contribute to pancreatic $\beta$-cellspecific silencing of monocarboxylate transporter 1 (Mct1). Mol Cell Biol. 2011;31(15):3182-3194.

10. Mendell JT, Olson EN. MicroRNAs in stress signaling and human disease. Cell. 2012;148(6):1172-1187.

11. Dumortier O, Hinault C, Van Obberghen E. MicroRNAs and metabolism crosstalk in energy homeostasis. Cell Metab. 2013;18(3):312-324

12. Lynn FC, et al. MicroRNA expression is required for pancreatic islet cell genesis in the mouse. Dia betes. 2007;56(12):2938-2945.

13. Melkman-Zehavi T, et al. miRNAs control insulin content in pancreatic $\beta$-cells via downregulation of transcriptional repressors. EMBO J. 2011; 30(5):835-845.

14. Mandelbaum AD, et al. Dysregulation of Dicer1 in $\beta$ cells impairs islet architecture and glucose metabolism. Exp Diabetes Res. 2012;2012:470302.

15. Poy MN, et al. miR-375 maintains normal pancreatic $\alpha$ - and $\beta$-cell mass. Proc Natl Acad Sci U S A 2009;106(14):5813-5818.

16. Joglekar MV, Joglekar VM, Hardikar AA. Expression of islet-specific microRNAs during human pancreatic development. Gene Expr Patterns. 2009; 9(2):109-113.

17. Bravo-Egana V, et al. Quantitative differential expression analysis reveals miR-7 as major islet microRNA. Biochem Biophys Res Commun. 2008;366(4):922-926.

18. Kredo-Russo S, Ness A, Mandelbaum AD, Walker MD, Hornstein E. Regulation of pancreatic microRNA-7 expression. Exp Diabetes Res. 2012; 2012:695214.

19. Tessmar-Raible K, et al. Conserved sensory-neurosecretory cell types in annelid and fish forebrain: insights into hypothalamus evolution. Cell. 2007;129(7):1389-1400.

20. Choudhury NR, et al. Tissue-specific control of brain-enriched miR-7 biogenesis. Genes Dev. 2013; 27(1):24-38.

21. Wang Y, Vogel G, Yu Z, Richard S. The QKI-5 and QKI-6 RNA binding proteins regulate the expression of microRNA 7 in glial cells. Mol Cell Biol. 2013;33(6):1233-1243.

22. Memczak S, et al. Circular RNAs are a large class of animal RNAs with regulatory potency. Nature. 2013;495(7441):333-338.

23. Hansen TB, et al. Natural RNA circles function as efficient microRNA sponges. Nature. 2013; 495(7441):384-388

24. Herrera PL. Adult insulin- and glucagon-producing cells differentiate from two independent cell lineages. Development. 2000;127(11):2317-2322.

25. Kanno T, Rorsman P, Göpel SO. Glucose-dependent regulation of rhythmic action potential firing in pancreatic $\beta$-cells by kATP-channel modulation. J Physiol. 2002;545(Pt 2):501-507.

26. Gopel S, Kanno T, Barg S, Galvanovskis J, Rorsman $P$. Voltage-gated and resting membrane currents recorded from B-cells in intact mouse pancreatic islets. J Physiol. 1999;521(pt 3):717-728.
27. Olofsson CS, et al. Fast insulin secretion reflects exocytosis of docked granules in mouse pancreatic B-cells. Pflugers Arch. 2002;444(1-2):43-51.

28. Burre J, et al. $\alpha$-Synuclein promotes SNAREcomplex assembly in vivo and in vitro. Science. 2010;329(5999):1663-1667.

29. Wang YW, et al. Spontaneous type 2 diabetic rodent models. J Diabetes Res. 2013;2013:401723.

30. Guo $S$, et al. Inactivation of specific $\beta$ cell transcription factors in type 2 diabetes. J Clin Invest. 2013;123(8):3305-3316

31. Kredo-Russo S, et al. Pancreas-enriched miRNA refines endocrine cell differentiation. Development. 2012;139(16):3021-3031

32. Gargani S, et al. Adaptive changes of human islets to an obesogenic environment in the mouse. Diabetologia. 2013;56(2):350-358.

33. Shibasaki T, et al. Essential role of Epac2/ Rap1 signaling in regulation of insulin granule dynamics by cAMP. Proc Natl Acad Sci U S A. 2007; 104(49):19333-19338.

34. Barg S, et al. Delay between fusion pore opening and peptide release from large dense-core vesicles in neuroendocrine cells. Neuron. 2002;33(2):287-299.

35. Wang Y, Liu J, Liu C, Naji A, Stoffers DA. MicroRNA-7 regulates the mTOR pathway and proliferation in adult pancreatic $\beta$-cells. Diabetes. 2013;62(3):887-895.

36. Spillantini MG, Goedert M. The alpha-synucleinopathies: Parkinson's disease, dementia with Lewy bodies, and multiple system atrophy. Ann NY Acad Sci. 2000;920:16-27.

37. Chandra S, Gallardo G, Fernandez-Chacon R, Schluter OM, Sudhof TC. $\alpha$-Synuclein cooperates with $\operatorname{CSP} \alpha$ in preventing neurodegeneration. Cell. 2005;123(3):383-396.

38. Steneberg $\mathrm{P}$, et al. The type 2 diabetes-associated gene ide is required for insulin secretion and suppression of $\alpha$-synuclein levels in $\beta$-cells. Diabetes. 2013;62(6):2004-2014.

39. Pinton P, et al. Dynamics of glucose-induced membrane recruitment of protein kinase $C \beta$ II in living pancreatic islet $\beta$-cells. J Biol Chem. 2002; 277(40):37702-37710.

40. Shu Y, Liu X, Yang Y, Takahashi M, Gillis KD. Phosphorylation of SNAP-25 at Ser187 mediates enhancement of exocytosis by a phorbol ester in INS-1 cells. J Neurosci. 2008;28(1):21-30.

41. Nili U, et al. Munc18-1 phosphorylation by protein kinase $\mathrm{C}$ potentiates vesicle pool replenishment in bovine chromaffin cells. Neuroscience. 2006; 2(143):487-500.

42. Barclay JW, et al. Phosphorylation of Munc18 by protein kinase $\mathrm{C}$ regulates the kinetics of exocytosis. J Biol Chem. 2003;278(12):10538-10545.

43. Esguerra JL, Bolmeson C, Cilio CM, Eliasson L. Differential glucose-regulation of microRNAs in pancreatic islets of non-obese type 2 diabetes model Goto-Kakizaki rat. PLoS One. 2011;6(4):e18613.

44. Andrali SS, Sampley ML, Vanderford NL, Ozcan S. Glucose regulation of insulin gene expression in pancreatic $\beta$-cells. Biochem J. 2008;415(1):1-10.

45. Harmon JS, Stein R, Robertson RP. Oxidative stressmediated, post-translational loss of MafA protein as a contributing mechanism to loss of insulin gene expression in glucotoxic beta cells.J Biol Chem. 2005;280(12):11107-11113.

46. Harmon JS, et al. $\beta$-Cell-specific overexpression of glutathione peroxidase preserves intranuclear MafA and reverses diabetes in $\mathrm{db} / \mathrm{db}$ mice. Endocri- nology. 2009;150(11):4855-4862.

47. Sander M, et al. Genetic analysis reveals that PAX6 is required for normal transcription of pancreatic hormone genes and islet development. Genes Dev. 1997;11(13):1662-1673

48. St-Onge L, Sosa-Pineda B, Chowdhury K, Mansouri A, Gruss P. Pax6 is required for differentiation of glucagon-producing $\alpha$-cells in mouse pancreas. Nature. 1997;387(6631):406-409.

49. Xuan S, et al. Pancreas-specific deletion of mouse Gata 4 and Gata 6 causes pancreatic agenesis. J Clin Invest. 2012;122(10):3516-3528.

50. Carrasco M, Delgado I, Soria B, Martin F, Rojas A. GATA4 and GATA6 control mouse pancreas organogenesis. J Clin Invest. 2012;122(10):3504-3515.

51. Hart AW, Mella S, Mendrychowski J, van Heyningen $\mathrm{V}$, Kleinjan DA. The developmental regulator Pax6 is essential for maintenance of islet cell function in the adult mouse pancreas. PLoS One. 2013;8(1):e54173.

52. Ketola I, et al. Transcription factor GATA-6 is expressed in the endocrine and GATA-4 in the exocrine pancreas. Mol Cell Endocrinol. 2004; 226(1-2):51-57.

53. De Franco E, et al. GATA6 mutations cause a broad phenotypic spectrum of diabetes from pancreatic agenesis to adult-onset diabetes without exocrine insufficiency. Diabetes. 2013;62(3):993-997.

54. Kopp JL, et al. Sox $9^{+}$ductal cells are multipotent progenitors throughout development but do not produce new endocrine cells in the normal or injured adult pancreas. Development. 2011; 138(4):653-665.

55. Talchai C, Xuan S, Lin HV, Sussel L, Accili D. Pancreatic beta cell dedifferentiation as a mechanism of diabetic $\beta$ cell failure. Cell. 2012;150(6):1223-1234.

56. Nagamatsu $S$, et al. Decreased expression of t-SNARE, syntaxin 1 , and SNAP-25 in pancreatic $\beta$-cells is involved in impaired insulin secretion from diabetic GK rat islets: restoration of decreased t-SNARE proteins improves impaired insulin secretion. Diabetes. 1999;48(12):2367-2373.

57. Gaisano HY, Ostenson CG, Sheu L, Wheeler MB, Efendic S. Abnormal expression of pancreatic islet exocytotic soluble $\mathrm{N}$-ethylmaleimide-sensitive factor attachment protein receptors in Goto-Kakizaki rats is partially restored by phlorizin treatment and accentuated by high glucose treatment. Endocrinology. 2002;143(11):4218-4226.

58. Ostenson CG, Gaisano H, Sheu L, Tibell A, Bartfai $\mathrm{T}$. Impaired gene and protein expression of exocytotic soluble $\mathrm{N}$-ethylmaleimide attachment protein receptor complex proteins in pancreatic islets of type 2 diabetic patients. Diabetes. 2006;55(2):435-440

59. Andersson SA, et al. Reduced insulin secretion correlates with decreased expression of exocytotic genes in pancreatic islets from patients with type 2 diabetes. Mol Cell Endocrinol. 2012;364(1-2):36-45.

60. Sudhof TC, Rizo J. Synaptic vesicle exocytosis. Cold Spring Harb Perspect Biol. 2011;3(12):a005637.

61. de Chevigny A, et al. miR-7a regulation of Pax6 controls spatial origin of forebrain dopaminergic neurons. Nat Neurosci. 2012;15(8):1120-1126.

62. Braun $M$, et al. Voltage-gated ion channels in human pancreatic $\beta$-cells: electrophysiological characterization and role in insulin secretion. Diabetes. 2008;57(6):1618-1628.

63. Barg S, et al. Fast exocytosis with few $\mathrm{Ca}(2+)$ channels in insulin-secreting mouse pancreatic B cells. Biophys J. 2001;81(6):3308-3323. 